\title{
Ausmaß und Entwicklung von Geschlechterunter- schieden im Bereich des kriminellen Verhaltens und der Kriminalitätswahrnehmung
}

\section{Dirk Baier}

\section{Aufbau des Beitrags}

Das kriminelle Verhalten gehört zu jenen Bereichen, für die Geschlechterunterschiede schon seit langem bekannt sind. So, wie sich für die meisten Gesellschaften und die meisten historischen Zeitpunkte die Alters-Kriminalitäts-Kurve als gültig erweist, gilt dies auch für den Geschlechterunterschied: Männer bzw. Jungen treten häufiger mit kriminellen Verhaltensweisen in Erscheinung als Frauen bzw. Mädchen, bei der Mehrheit der Delikte, in der Mehrheit der Gesellschaften und während verschiedener historischer Epochen. Anliegen dieses Beitrags soll es jedoch nicht sein, die Ursachen für den ,gender gap“ in der Kriminalität zu untersuchen (vgl. hierfür u. a. Baier, 2011c; Junger-Tas, Ribeaud \& Cruyff, 2004; Scheithauer, 2003). Dabei wäre eine Vielzahl an Faktoren zu betrachten, angefangen von biologisch-genetischen Faktoren, über sozialisationsspezifische hin zu gesellschaftlichen bzw. kulturellen Faktoren. Diese Bandbreite an Faktoren kann mit vorliegenden empirischen Daten nicht adäquat abgebildet werden. Anliegen dieses Beitrages ist es stattdessen, das Ausmaß und die Entwicklung des ,gender gap“ für Deutschland für verschiedene Altersgruppen für die zurückliegenden Jahre zu beschreiben. Dabei sollen Antworten auf folgende Fragen gegeben werden:

1. In welchen Deliktsbereichen sind in Deutschland die Abstände zwischen Männern und Frauen besonders groß, in welchen eher klein? Finden sich Geschlechtsunterschiede im Jugend- wie im Erwachsenenalter?

2. Hat es in den letzten Jahren eine Angleichung der Geschlechter im kriminellen Verhalten gegeben oder fällt in bestimmten Deliktsbereichen der Abstand mittlerweile noch größer aus als früher? Ist also auch im Bereich des kriminellen Verhaltens eine „Krise der Jungen“ erkennbar? 
Verlässliche Antworten auf diese Fragen zu finden, setzt voraus, dass es verlässliche Datenquellen zum kriminellen Verhalten der Bevölkerung gibt. Dies ist jedoch nicht der Fall. Im Wesentlichen lassen sich zwei Informationsquellen unterscheiden, mit jeweils spezifischen Nachteilen. Die erste ist die sog. Hellfeldstatistik, in der registrierte Straftaten (inkl. Täter und Opfer) ausgewiesen werden; die bekannteste Statistik hier ist die Polizeiliche Kriminalstatistik. Der Nachteil dieser Statistik ist, dass nur angezeigte oder anderweitig der Polizei zur Kenntnis gelangte Straftaten gezählt und berichtet werden. Ein je nach Deliktsbereich unterschiedlich großer Anteil des kriminellen Verhaltens wird nicht entdeckt, nicht angezeigt und verbleibt damit im sog. Dunkelfeld. Die Polizeiliche Kriminalstatistik weist also immer nur einen mehr oder weniger großen Anteil der Kriminalität aus. Nachteilig ist dabei zusätzlich, dass das Verhältnis zwischen dem Hell- und dem Dunkelfeld nicht nur mit dem Delikttyp, sondern auch über die Zeit bzw. über bestimmte geographische Einheiten hinweg variiert. Ansteigende oder rückläufige Entwicklungen in der Hellfeldstatistik ebenso wie Unterschiede zwischen Städten, Regionen oder Bundesländern können deshalb sowohl tatsächliche Veränderungen bzw. Unterschiede markieren; sie können aber auch auf Veränderungen bzw. Unterschieden im Anzeigeverhalten, in den polizeilichen Ermittlungsaktivitäten, in der Registrierungspraxis, in gesetzlichen Rahmenbedingungen usw. basieren (vgl. Lamnek, 1998, S. 384ff).

Diese Nachteile können mit Dunkelfeldbefragungen, der zweiten zentralen Informationsquelle, weitestgehend behoben werden. Die Bezeichnung ist etwas irreführend, weil diese Studien nicht nur beanspruchen, den nicht der Polizei zur Kenntnis gelangten Anteil der Kriminalität zu erfassen, sondern sowohl die Hell- als auch die Dunkelfeldkriminalität. Dunkelfeldstudien sind i. d. R. so angelegt, dass eine repräsentative Auswahl der Bevölkerung bzw. einer Bevölkerungsgruppe über ihre Erlebnisse mit kriminellen Verhaltensweisen befragt wird. Mindestens drei Nachteile sind jedoch auch für diese Herangehensweise zu nennen: Erstens ist es nicht möglich, die gesetzlich definierten Straftaten für alle Befragten gleichermaßen verständlich in Deliktsbeschreibungen in Fragebögen zu übersetzen. Ein von einem Befragten erlebter Übergriff mag von diesem bspw. als Raub eingestuft werden, obwohl es strafrechtlich betrachtet nur ein Diebstahl war. Befragte klassifizieren ihre Erlebnisse in subjektiver Weise und orientieren sich nur teilweise an objektiven Kriterien. Ein zweiter Nachteil ist, dass eine exakte Repräsentativität von Stichproben nie erreicht werden kann. Gerade jene Bevölkerungsgruppen, die besonders viele kriminelle Erfahrungen haben, werden nicht willens oder nicht fähig 
sein, sich an einer Befragung zu beteiligen. Drittens gibt es Straftaten, die über Bevölkerungsbefragungen kaum valide abgebildet werden können, z. B. die Wirtschafts- oder organisierte Kriminalität. Dies ist deshalb der Fall, weil es sich teilweise um opferlose Taten oder Taten in einem wissenschaftlich nicht zugänglichen Feld handelt. Auch für andere sehr sensible Themenbereiche wie z. B. das Thema der Vergewaltigung in der Ehe ist anzunehmen, dass es ein Dunkelfeld des Dunkelfeldes gibt, dass also Dunkelfeldbefragungen hier nur begrenzt Informationen liefern, insofern nicht alle Opfer Auskunft über ihre Erlebnisse erteilen möchten.

Im Vergleich zur Polizeilichen Kriminalstatistik erweist sich der Bereich der Dunkelfeldforschung in Deutschland in einer weiteren Hinsicht als problematisch: Während die Polizeiliche Kriminalstatistik jährlich bis in die 1950er-Jahre hinein vorliegt (seit 1993 bundesweit einheitlich), existiert keine kontinuierliche Dunkelfeldforschung wie dies in Ländern wie den USA oder Großbritannien der Fall ist. Nur für wenige Kriminalitätsbereiche bzw. Altersgruppen gibt es deutschlandweit repräsentative Dunkelfeldbefragungen; und nur sehr selten wurde diese in vergleichbarer Weise wiederholt, so dass auf dieser Basis Entwicklungsaussagen (im Geschlechtervergleich) möglich sind.

In Bezug auf Jugendliche erfolgte die erste deutschlandweit repräsentative Befragung, in der sowohl Täterschaften als auch Opererfahrungen erhoben wurden, erst in den Jahren 2007/2008 durch das Kriminologische Forschungsinstitut Niedersachsen (KFN); Ergebnisse dieser Befragung sollen nachfolgend in Bezug auf den Geschlechtervergleich vorgestellt werden. Der Großteil der bis dato vorhandenen Jugendbefragungen weist einen lokalen Bezug auf, konzentriert sich also auf einzelne Städte oder Landkreise (u. a. Wetzels, Enzmann, Mecklenburg \& Pfeiffer, 2001; Dünkel, Gebauer \& Geng, 2007; Baier, Pfeiffer, Windzio \& Rabold, 2006). Wiederholungsbefragungen in einigen ausgewählten Gebieten ermöglichen hier jedoch, die Entwicklung des kriminellen Verhaltens im Geschlechtervergleich nachzuzeichnen, worauf ebenfalls eingegangen werden soll.

Die Dunkelfeldstudien unter Erwachsenen konzentrieren sich fast ausschließlich auf die Erfassung von Opferschaften. Täterschaften wurden in der Vergangenheit nur im ALLBUS in Bezug auf niedrigschwellige Taten wie das Schwarzfahren (vgl. Baier \& Branig, 2009), in einzelnen Städten (vgl. Mehlkop, 2011 für Dresden) oder für einzelne Altersgruppen (vgl. Kunz, 2011 für ab 49jährige Personen) untersucht; hier ist die Forschungslage daher vollkommen unzureichend, weshalb keine Dunkelfeldbefunde vorgestellt werden sollen. Studien zu Viktimisierungserfahrungen finden 
sich hingegen deutlich häufiger (vgl. für einen Überblick u. a. Rabold, 2014). Eine erste deutschlandweit repräsentative Studie stellt der International Crime Survey von 1988 dar, in dessen Rahmen in Deutschland 5,000 Personen zu ihren Opfererfahrungen befragt wurden (Kury, 1992). Erwähnenswert ist darüber hinaus eine Opferbefragung, die in den Jahren 1997 und 1998 unter mehr als 23,000 Befragten durchgeführt wurde (Heinz, Spieß, Schnell \& Kreuter, 1998). Auch am KFN wurden in der Vergangenheit Viktimisierungsbefragungen durchgeführt. Eine erste Befragung unter 15,000 Personen erfolgte 1992 unter ab 16-Jährigen (vgl. Bilsky, Mecklenburg \& Wetzels, 1992). Der Schwerpunkt dieser Befragung lag allerdings im Bereich der Viktimisierung mit sexueller Gewalt (Wetzels, 1997). Diese Befragung wurde im Jahr 2011 zum zweiten Mal unter 16- bis 40-jährigen Personen durchgeführt (Stadler, Bieneck \& Pfeiffer, 2012). Viktimisierungserfahrungen wurden darüber hinaus in einer in den Jahren 2004, 2006 und 2010 vergleichbar durchgeführten KFNBefragung erfasst (vgl. Baier et al., 2011). Auf Basis dieser KFN-Opferbefragungen sollen nachfolgend Aussagen zur Viktimisierungshäufigkeit im Geschlechtervergleich für Erwachsene vorgestellt werden.

Vorangestellt werden den Auswertungen von Dunkelfeldbefragungen von Jugendlichen und Erwachsenen jeweils entsprechende Auswertungen der Polizeilichen Kriminalstatistik, deren Gültigkeit mit den Dunkelfelddaten partiell geprüft werden kann. Zunächst soll dabei der Bereich der Gewaltkriminalität beleuchtet werden, anschließend dann der Bereich der Eigentumskriminalität, der in Dunkelfeldstudien weniger intensiv untersucht wird. Die Analysen werden zusätzlich um drei Exkurse erweitert: Erstens werden Geschlechterunterschiede im Bereich der Kriminalitätserfahrungen in spezifischen Bevölkerungsgruppen berichtet, die in vergangenen Forschungsprojekten des KFN im Fokus standen: Polizeibeamte und Strafgefangene. $\mathrm{Zu}$ vermuten ist, dass die Geschlechterunterschiede bei beiden Gruppen geringer als in der Allgemeinbevölkerung ausfallen. Ein zweiter Exkurs bezieht sich auf Bereiche aggressiven Verhaltens, die im Strafrecht weitestgehend unberücksichtigt sind: verbale und relationale Aggressionen. Für diesen nicht-physischen Gewaltbereich wird ebenfalls verschiedentlich berichtet, dass Geschlechterunterschiede nicht vorhanden sind oder sich sogar ins Gegenteil verkehren (u. a. Scheithauer, 2003). Zuletzt soll der Blick auf Einstellungen bzgl. des Themas Kriminalität gerichtet werden. Für die Furcht vor Kriminalität oder die Wahrnehmung, dass Kriminalität immer weiter ansteigt, lassen sich wiederum geringere Geschlechterunterschiede vermuten als für konkretes Verhalten. Anliegen der Exkurse ist es damit, ein umfassenderes Bild des Zusammenhangs von 
Geschlecht und Kriminalität zu zeichnen und auf Bereiche aufmerksam zu machen, in denen die Unterschiede zwischen den Geschlechtern weit weniger deutlich ausfallen.

\section{Gewaltkriminalität}

\subsection{Jugendliche als Täter}

\section{Polizeiliche Kriminalstatistik}

Im Jahr 2011 wurden bundesweit 31,730 Personen im Alter zwischen 14 und unter 18 Jahren wegen des Begehens eines Gewaltkriminalitätsdelikts polizeilich als Täter registriert. Bezogen auf die Gesamtzahl an Jugendlichen in Deutschland (3.2 Mio.) bedeutet dies, dass ein Prozent aller Jugendlichen polizeilich auffällig geworden ist. Als Gewaltkriminalität werden dabei folgende Delikte definiert: Mord/Totschlag, Vergewaltigung, Raub, gefährliche bzw. schwere Körperverletzung. Werden zusätzlich vorsätzliche, leichte Körperverletzungen einbezogen, so erhöht sich der Anteil an Tatverdächtigen auf 2.0\% (2011: 30,935 Tatverdächtige). Bei männlichen Jugendlichen liegt dieser Anteil mit 3.0\% (Gewaltkriminalität: $1.6 \%$, vorsätzliche, leichte Körperverletzung: $1.4 \%$ ) mehr als dreimal so hoch wie bei Mädchen, bei denen der Anteil $0.8 \%$ beträgt $(0.4$ bzw. $0.5 \%$ ). Da der Mord/Totschlag bzw. die Vergewaltigung zahlenmäßig im Jugendalter $\mathrm{zu}$ vernachlässigende Delikte darstellen - 2011 wurden deutschlandweit 167 Jugendliche des Mords/Totschlags, 703 Jugendliche der Vergewaltigung verdächtigt - wird sich nachfolgend bei der Betrachtung von Entwicklungstrends auf die anderen drei genannten Delikte beschränkt.

Die Entwicklung wird dabei anhand der Tatverdächtigenbelastungszahl nachgezeichnet. Diese gibt an, wie viele Jugendliche pro 100,000 als Tatverdächtige registriert wurden; geteilt durch 1,000 entspricht sie einer gewöhnlichen Prozentzahl. In Abbildung 1 sind die Belastungszahlen für drei Jahre im Vergleich der Geschlechter dargestellt. Das Jahr 1997 wurde als Ausgangspunkt gewählt, weil sich die im nächsten Abschnitt vorgestellten KFN-Schülerbefragungen ebenfalls bei der ersten Erhebung auf dieses Jahr beziehen. Werden zunächst die Trends betrachtet, so ergibt sich für männliche wie für weibliche Jugendliche, dass der Raub seit 1997 kontinuierlich rückläufig ist. Körperverletzungen sind dagegen zunächst deutlich gestiegen. In jüngerer Zeit fallen die Belastungszahlen allerdings 
wieder, zumindest bei der gefährlichen/schweren Körperverletzung. Im Großen und Ganzen kann also gefolgert werden, dass die Jugendgewaltkriminalität in Deutschland zumindest in der kurzfristigen Betrachtung rückläufig ist (vgl. auch Baier, 2011b).

Im Geschlechtervergleich zeigt sich für beide Formen der Körperverletzungen eine Annäherung der Belastungszahlen. Während die Belastungszahl der Jungen für gefährliche/ schwere Körperverletzung im Jahr 1997 die der Mädchen noch um das Fünffache überschritt, war es 2011 nur mehr das Vierfache. Bei weiblichen Jugendlichen ist ein stärkerer Anstieg der Belastungszahlen auszumachen, in dessen Folge sich die Zahlen langsam annähern. Für Raubdelikte ist ein vergleichbarer Trend nicht zu identifizieren; das Verhältnis liegt unverändert bei etwa eins zu neun.

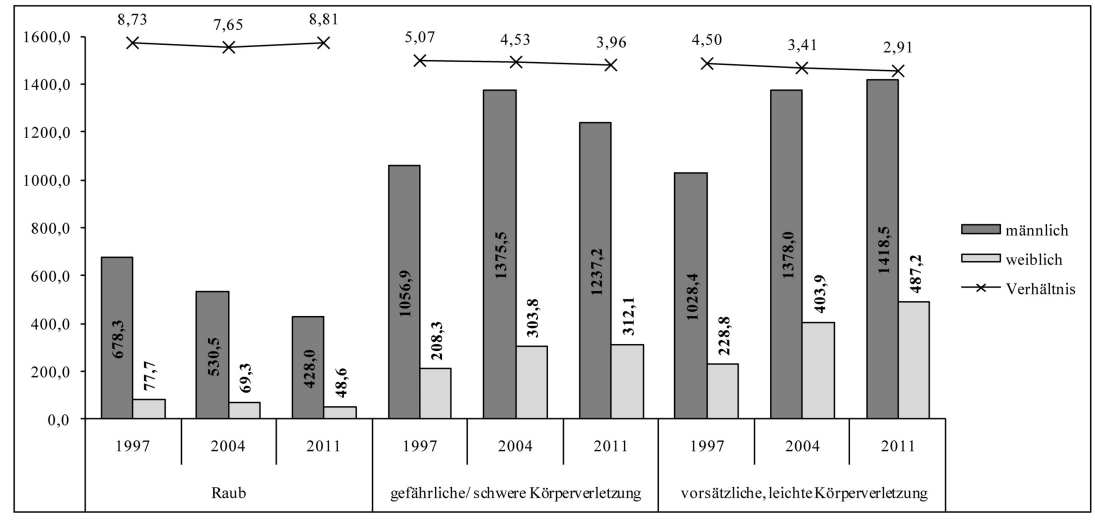

Abbildung 1. Tatverdächtigenbelastungszahl Jugendlicher für verschiedene Gewaltdelikte nach Geschlecht und Jahr.

Dunkelbefragungen

Die Betrachtung von Dunkelfelddaten ergibt in dreifacher Hinsicht ein von der Polizeilichen Kriminalstatistik abweichendes Bild:

1. Die Prävalenzraten des Gewaltverhaltens liegen deutlich höher.

2. Eine rückläufige Entwicklung des Gewaltverhaltens setzt bereits deutlich früher ein.

3. Jungen und Mädchen nähern sich über die Jahre hinweg auch im Bereich der Körperverletzungen nicht an. 
Die Gewalt-Prävalenzraten lassen sich am verlässlichsten anhand der deutschlandweit repräsentativen Schülerbefragung 2007/2008 bestimmen (Baier, Pfeiffer, Simonson \& Rabold, 2009). Zwar wurden die Datenerhebungen ausschließlich in der neunten Jahrgangsstufe durchgeführt, zumindest die 14- bis 16-jährigen werden damit aber recht gut erreicht. Insgesamt wurden im Rahmen dieser Studie bei einer Rücklaufquote von 62.1\% 44,610 Neuntklässler befragt. Das Begehen einer Raubtat geben in Bezug auf die zurückliegenden zwölf Monate 2.5\% der Befragten an; diese Rate liegt etwa fünfmal höher als im Hellfeld. Übereinstimmend mit dem Hellfeld existiert ein großer Unterschied zwischen den Geschlechtern: Jungen haben zu 4.0\%, Mädchen zu 1.0\% Raubtaten ausgeführt. Dass der Abstand niedriger ist als im Hellfeld, könnte damit in Zusammenhang stehen, dass von den Befragten auch Taten geringerer Schwere berichtet werden, die von der Polizei nicht als Raubtaten eingestuft werden. Bei den schweren Körperverletzungen liegt die Prävalenzrate im Dunkelfeld bei 2.9\% (Jungen: 4.9\%, Mädchen: 0.9\%), bei den leichten Körperverletzungen bei 11.7\% (Jungen: 17.8\%, Mädchen: 5.5\%). Auch bei diesen Delikten gilt damit, dass im Dunkelfeld die Raten mindestens ca. dreimal so hoch wie im Hellfeld liegen; der Abstand zwischen den Geschlechtern entspricht aber den Hellfeld-Verhältnissen.

Die Erklärung für die im Dunkelfeld höheren Prävalenzraten ist im Anzeigeverhalten zu suchen. Nur ein Teil der jugendlichen Gewaltkriminalität gelangt über eine Anzeige in die Kriminalstatistik. Entsprechend der Befunde der Schülerbefragung 2007/2008 liegt die Anzeigequote bei leichten Körperverletzungen mit $18.9 \%$ am niedrigsten. Bei schweren Körperverletzungen (36.8\%) und bei Raubtaten (40.2\%) ist sie hingegen etwa doppelt so hoch.

Bezüglich der Entwicklung der Jugendgewalt im Dunkelfeld existieren bislang nur für einzelne Gebiete, nicht für die gesamte Bundesrepublik Befunde. Eine in den Jahren 1998 bzw. 2005/2006 wiederholt durchgeführte Schülerbefragung in vier Städten (München, Stuttgart, Hannover, Schwäbisch Gmünd) erlaubt aufgrund der hohen Fallzahlen eine auch nach dem Geschlecht differenzierende Auswertung. Die Prävalenzrate für den Raub ist in diesem Beobachtungszeitraum von 4.4 auf 3.2\% signifikant gefallen (Baier, 2008, S. 26), was dem Trend im Hellfeld entspricht. Entgegen den Hellfelddaten ist aber auch die leichte Körperverletzung zurückgegangen: 1998 gaben noch 18.4\% der Befragten an, eine solche Körperverletzung verübt zu haben, 2005/2006 waren es nur noch 15.8\%. Schwere Körperverletzungen wurden aus Täterperspektive nicht erhoben. Für zwei weitere Gewalttaten, die Erpressung und die Bedrohung mit 
Waffen, ergibt sich aber ebenfalls ein Rückgang der Prävalenzraten, weshalb davon auszugehen ist, dass sich eine vergleichbare Entwicklung auch für schwere Körperverletzungen gezeigt hätte.

Die insbesondere zu den Körperverletzungen diskrepante Entwicklung im Vergleich mit der Kriminalstatistik lässt sich damit erklären, dass die Anzeigebereitschaft gestiegen ist. Aus einem kleiner werdenden Dunkelfeld wird also ein überproportionaler Anteil der Gewaltkriminalität über eine Anzeigeerstattung im Hellfeld sichtbar. Bei leichten Körperverletzungen ist die Anzeigequote bspw. von 14.8 auf 19.7\% gestiegen (Baier, 2008, S. 19), bei schweren Körperverletzungen von 21.6 auf $23.5 \%$; die Anzeigebereitschaft wurde auf Basis der Angaben der Opfer bestimmt und aus Opferperspektive wurden schwere Körperverletzungen (bzw. Körperverletzungen mit Waffen) ebenfalls erhoben. Auch beim Raub hat es eine Zunahme der Anzeigequote gegeben (von 34,3 auf 49,4 \%; Baier, 2008, S. 18).

Von besonderem Interesse sind nun die geschlechtsspezifischen Entwicklungstrends. In Abbildung 2 sind diese erstens für die Gewaltprävalenzraten (Täterschaft) und zweitens für die Anzeigequoten abgetragen. Für den Raub ergibt sich dabei eine Vergrößerung des „gender gap“: Jungen haben in der neueren Befragung 4.0-mal häufiger eine Raubtat ausgeführt, 1998 lag die Rate nur 3.6-mal höher. Sowohl bei Jungen als auch bei Mädchen ist aber die Prävalenzrate für Raub gefallen, für Mädchen jedoch stärker als für Jungen. Im Hellfeld zeichnete sich eine Konstanz des Geschlechterverhältnisses ab. Auch in Bezug auf die leichten Körperverletzungen widersprechen die Befunde der wiederholt durchgeführten Schülerbefragung den Hellfeldbefunden: Das Verhältnis zwischen den Raten der Jungen und denen der Mädchen ist hier konstant geblieben und nicht gesunken; d. h. der Rückgang in den Täterraten vollzieht sich bei beiden Geschlechtern im selben Tempo. Für die zur Hellfeldentwicklung abweichenden Befunde kann einmal mehr das Anzeigeverhalten als Erklärungsfaktor benannt werden. Am Beispiel der Körperverletzung ist erkennbar, dass sowohl die Anzeigebereitschaft bei männlichen wie bei weiblichen Tätern gestiegen ist. Bei weiblichen Tätern hat sich aber die Anzeigequote fast verdoppelt (von 16.4 auf 29.0\%). Dies bedeutet, dass überproportional viel von weiblichen Jugendlichen ausgeübte Gewalt ins Hellfeld kommt. Daraus darf aber nicht der Schluss gezogen werden, dass Mädchen in ihrem Verhalten den Jungen immer ähnlicher würden; eher das Gegenteil ist nach den Dunkelfeldbefunden der Fall. Es hat sich stattdessen die Toleranz gegenüber der von Mädchen ausgeübten Gewalt verändert. Täterinnen müssen heute häufiger als Täter damit rechnen, polizei- 
lich registriert zu werden. Einschränkend ist darauf hinzuweisen, dass diese Befunde nur anhand weniger Städte erarbeitet werden konnten, eine Generalisierung auf die gesamte Bundesrepublik ist nur bedingt möglich.

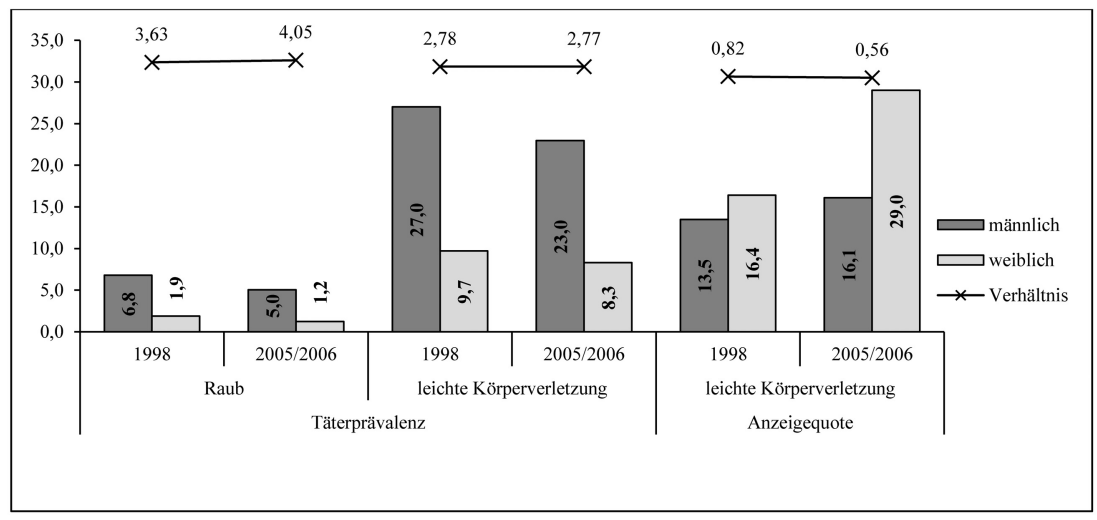

Abbildung 2. Entwicklung der Gewaltprävalenzraten sowie der Anzeigequote nach Geschlecht und Jahr (in \%).

\subsection{Opferschaften in der Erwachsenenbevölkerung}

\section{Polizeiliche Kriminalstatistik}

Die Viktimisierung von Erwachsenen durch Gewaltkriminalität lässt sich mit der Kriminalstatistik im Geschlechtervergleich anhand der Opfergefährdungszahl darstellen. Diese Zahl gibt an, wie viel Personen pro 100,000 einer Altersgruppe Gewaltübergriffe erleben mussten (und angezeigt haben). An dieser Stelle sollen als Erwachsene die Altersgruppe der 21- bis 60-Jährigen betrachtet werden. Wie Abbildung 3 zeigt, besteht das höchste Risiko der Gewaltopferschaft hinsichtlich der vorsätzlichen, leichten Körperverletzung, die hier erneut bei der Betrachtung der Gewaltdelikte einbezogen werden. Im Jahr 2011 haben 0.6\% der Erwachsenen eine solche Körperverletzung erlebt (271,961 Opfer bei einer Bevölkerungszahl von 44.2 Mio.). Der Mord bzw. Totschlag wurde hingegen nur von $0.004 \%$ erlebt, Versuche mit berücksichtigt (1,803 Opfer). Wird die Entwicklung der Opfergefährdungszahl betrachtet, so ergibt sich im Wesentlichen für beide Formen der Körperverletzungen ein Anstieg. Die vorsätzliche, leichte Körperverletzung hat sich seit 1995 in etwa verdoppelt. In Abbildung 3 ist für die erste Beobachtung nicht immer dasselbe Jahr her- 
angezogen worden, sondern z. T. das Jahr 1993, z. T. das Jahr 1995 bzw. 1998. Dies ist deshalb der Fall, weil nicht für alle Delikte seit 1993 Opfergefährdungszahlen getrennt für Männer und Frauen vorliegen. Es wurde dann jenes Jahr dargestellt, für das erstmalig verlässliche Zahlen vorhanden sind. Dass hier im Gegensatz zu den Jugendlichen nicht das Jahr 1997 als Ausgangspunkt der Betrachtung gewählt wurde, ist darauf zurückzuführen, dass die KFN-Opferbefragungen z. T. bis ins Jahr 1992 zurückreichen. Für den Raub ist ähnlich wie bei den Jugendlichen ein Rückgang der Zahlen festzustellen, ebenso beim Mord/Totschlag. Bei der Vergewaltigung ist die aktuelle Opfergefährdungszahl vergleichbar der Zahl von 1998.

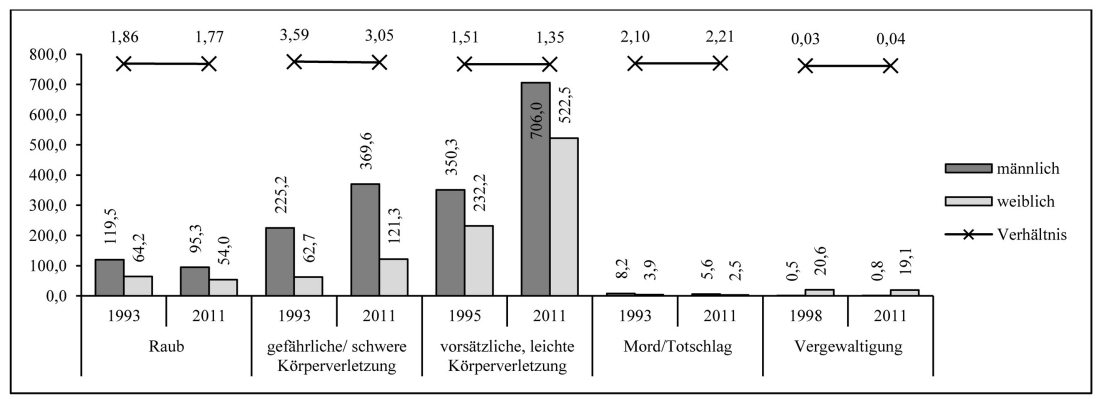

Abbildung 3. Opfergefährdungszahl Erwachsener (21- bis 60-Jähriger) für verschiedene Gewaltdelikte nach Geschlecht und Jahr.

Die Trends sind dabei für männliche und weibliche Erwachsene ähnlich. Gleichwohl fallen sie vor allem im Bereich der Körperverletzungen bei den Frauen stärker aus, so dass sich die Opfergefährdungszahlen annähern. Beim Mord/Totschlag gehen die Zahlen geringfügig auseinander, d. h. für Frauen geht die Gefährdungszahl etwas stärker zurück als für Männer. Wird zuletzt der Bereich der Vergewaltigung betrachtet, ergibt sich ebenfalls eine Annäherung der Geschlechter, dieses Mal unter anderen Vorzeichen. Frauen erleben Vergewaltigungen deutlich häufiger als Männer. Ein Anstieg der Opfergefährdungszahl ist aber nur bei den Männern festzustellen, so dass der Geschlechterunterschied in diesem Deliktsbereich kleiner wird. Grundsätzlich ist bei all den genannten Entwicklungstrends ebenso wie bei den geschlechtsspezifischen Trends zu beachten, dass es sich um Entwicklungen im Hellfeld handelt, die auch durch Veränderungen im Anzeigeverhalten bedingt sein können und damit keine echten Veränderungen wiederspiegeln müssen 


\section{Dunkelfeldbefragung}

Um die Entwicklung von Gewaltopferschaften im Dunkelfeld zu untersuchen, kann auf zwei wiederholt durchgeführte KFN-Befragungen zurückgegriffen werden. Zum einen ist dies eine deutschlandweite Repräsentativbefragung der ab 16-jährigen, deutschen Wohnbevölkerung aus den Jahren 2004, 2006 und 2010 (vgl. Baier et al., 2011). Die Stichprobengröße schwankte zwischen 1,110 und 3,255 Befragten, die Rücklaufquoten lagen bei mindestens 64\%, was aufgrund der methodischen Anlage der Befragungen als Access-Panels nicht überrascht. Zum anderen kann der in den Jahren 1992 und 2011 durchgeführte Victimsurvey herangezogen werden (vgl. Bilsky et al., 1992; Stadler et al., 2012). Im Jahr 1992 erfolgte dieser unter ab 16-jährigen Personen mit deutscher Staatsangehörigkeit. Im Jahr 2011 wurden auch türkische und russischstämmige Befragte sowie nur Personen im Alter zwischen 16 und 40 Jahren einbezogen. Für die Auswertungen werden die Stichproben hinsichtlich der Merkmale Alter und Herkunft kompatibel gemacht, so dass im Jahr 2011 9,175 Befragte zur Verfügung stehen, im Jahr 1992 5,881 Befragte. Hinsichtlich der Auswertungen zum sexuellen Missbrauch in der Kindheit reduziert sich die Befragtenanzahl des Victimsurveys 1992 auf 2,098 Befragte, weil die entsprechenden Fragen nur einem Teil aller Befragten vorgelegt wurden.

Der Raub wurde nur in der Repräsentativbefragung und hier nur in den beiden Jahren 2004 und 2006 erhoben (Tabelle 1). In Übereinstimmung mit der Hellfeldentwicklung sinkt die 5-Jahres-Prävalenzrate von 2.2 auf 2.0\%. Diese Rate gibt an, welcher Anteil an Befragten im 5-Jahres-Zeitraum vor der Befragung mindestens ein Delikt erlebt hat. Es wird an dieser Stelle nicht die Zwölf-Monats-Prävalenz betrachtet, weil die Raten sehr niedrig ausfallen und letztlich nur von sehr wenigen Befragten, die ein Delikt erlebt haben, bestimmt würden. Wenn Zwölf-MonatsPrävalenzraten ermittelt werden sollen, müsste der Stichprobenumfang noch einmal deutlich vergrößert werden. Beim Raub zeigt sich zusätzlich, dass die Prävalenzraten nur bei den weiblichen Befragten fallen, was dazu führt, dass das Verhältnis der Raten männlicher und weiblicher Befragter ansteigt. Anzeigequoten können zur Repräsentativbefragung nicht berichtet werden, weil erst ab dem Jahr 2010 eine Erfassung der Anzeigeerstattung erfolgte.

Auch für den Bereich der Körperverletzungen ergibt sich unter Rückgriff auf die Repräsentativbefragung ein Rückgang der Prävalenzrate (von 4.6 auf 3.0\%), was der Hellfeldentwicklung deutlich widerspricht. Für 
Frauen wie für Männer fällt der Rückgang vergleichbar hoch aus, so dass das Verhältnis der Opferraten im Vergleich der Jahre 2004 und 2010 konstant bleibt. $\mathrm{Ob}$ die widersprüchliche Entwicklung zur Kriminalstatistik anzeigebedingt ist, lässt sich mit den Daten der Repräsentativbefragung nicht untersuchen, da hier, wie angesprochen, die Abfrage nach der Anzeigeerstattung erst ab dem Jahr 2010 erfolgte.

Aussagen zur Entwicklung der Anzeigequote erlaubt, mit einigen Einschränkungen, der Victimsurvey. Für die Körperverletzungen wird dabei, wie in den Schülerbefragungen, ein Anstieg der Anzeigequote von 24.2 auf $30.3 \%$ ausgewiesen. Zwei Punkte sind in Bezug auf die Anzeigequote aber zu beachten: Erstens bezieht sich die Anzeigequote zur Befragung 1992 auf Vorfälle, die im Jahr vorher stattgefunden haben; die Anzeigequote zur Befragung 2011 auf den letzten Vorfall innerhalb eines FünfJahres-Zeitraums. Zweitens wurden die Körperverletzungen zu beiden Zeitpunkten in unterschiedlicher Weise erfasst: 1992 wurde zwischen Körperverletzungen mit und Körperverletzungen ohne Waffen unterschieden; in der Befragung 2011 wurde nur nach Körperverletzungen gefragt, wobei im Fragetext nicht explizit ein Hinweis darauf gegeben wurde, dass diese mit oder ohne Waffen erfolgen können (Fragetext: „Sind Sie schon einmal absichtlich geschlagen, getreten, verprügelt, gestoßen, gewürgt oder auf andere Weise tätlich angegriffen worden?"). Möglich ist daher, dass Befragte auch jene Körperverletzungen angegeben haben, die mit Waffen erfolgt sind. Für das Jahr 1992 werden deshalb zwei Prävalenzraten ausgegeben: Die erste Prävalenzrate bezieht sich auf Körperverletzungen ohne Waffen, die zweite auf Körperverletzungen mit und ohne Waffen. Die Entwicklung fällt beide Male gleich aus: Der Anteil an Befragten, die in den zurückliegenden fünf Jahren Körperverletzungen erleben mussten, ist deutlich angestiegen. Dies entspricht dem Trend im polizeilichen Hellfeld. Dass die Raten einerseits deutlich höher liegen als in der Repräsentativbefragung ist damit zu erklären, dass nur 16- bis 40-Jährige betrachtet werden und nicht das gesamte Altersspektrum; jüngere Personen unterliegen einem erhöhten Risiko der Gewaltopferschaft. Dass der Trend andererseits gegenteilig zum Trend der Repräsentativbefragung ausfällt, könnte damit zusammenhängen, dass der betrachtete Zeitraum deutlich größer ist. Die Repräsentativbefragung macht eher kurzfristige Entwicklungen sichtbar, der Victimsurvey langfristige Entwicklungen. Der Victimsurvey bestätigt zudem den Trend des Hellfelds, dass die Prävalenzraten bei Frauen stärker steigen als bei Männern und dass insofern eine allmähliche Annäherung der Raten stattfindet. 
Dies ist auch im Bereich der Vergewaltigungen der Fall: Männer und Frauen nähern sich in ihrer Prävalenzrate an, was ebenso im Polizeilichen Hellfeld beobachtet werden konnte. Erneut werden für das Jahr 1992 zwei Prävalenzraten berichtet. Die erste bezieht sich auf die primäre Abfrage sexueller Gewalterfahrungen im Fragebogen (Fragetext: „Hat Sie schon einmal jemand mit Gewalt oder unter Androhung von Gewalt gegen Ihren Willen zum Beischlaf oder zu beischlafähnlichen Handlungen gezwungen oder versucht, das zu tun?"). Bei dieser zeigt sich ein Rückgang der Prävalenzrate von 1.7 auf 1.2\%. Dieser Rückgang betrifft in erster Linie die weiblichen Befragten, deren Rate von 3.1 auf $2.2 \%$ gefallen ist. In der Befragung 2011 wurde vor dem Fragetext explizit darauf hingewiesen, dass der nachfolgende Fragebogenteil ,sexuelle Gewalt außerhalb und innerhalb der Partnerschaft" erfasst. Dieser Hinweis fehlte in der Befragung von 1992, weshalb die Befragten möglicherweise seltener beim Antworten an die innerfamiliäre Vergewaltigung gedacht haben. An anderer Stelle des Fragebogens wurde sie aber noch einmal explizit erhoben (Fragetext: „Hat Sie schon einmal jemand, mit dem Sie verwandt sind oder mit dem Sie zusammen gelebt haben [...] zum Beischlaf [...] gezwungen [...]?“). In die zweite Prävalenzrate wurden die entsprechenden Antworten einbezogen; der Rückgang fällt dann noch einmal deutlicher aus, von 2.7 auf $1.2 \%$.

Wird für die Vergewaltigung die Entwicklung der Anzeigequote betrachtet, ergibt sich ein Rückgang von 22.2 auf 16.9\% (wobei männliche Opfer 1992 wie 2011 überhaupt nicht angezeigt haben). Dieser Befund ist aber in zweifacher Hinsicht zurückhaltend zu interpretieren: Erstens dürfte in der Anzeigequote von 1992 die innerfamiliäre Vergewaltigung seltener berücksichtigt worden sein; für diese ist die Anzeigequote aber besonders niedrig. Zweitens gehen 1992 nur sehr wenige Fälle in die Auswertungen ein, da die Anzeigequote für Übergriffe des Jahres 1991 erfragt wurde; die Fallzahl des Jahres 2011 ist deutlich höher. Aus anderen Dunkelfeldstudien ist bekannt, dass die Anzeigequote auch im Bereich der sexuellen Gewalt gestiegen ist (vgl. Baier, 2008).

Als ein letztes Delikt ist in Tabelle 1 der sexuelle Kindesmissbrauch dargestellt. Die Anzeigequote für das Jahr 1992 lässt sich hier nicht bestimmen, da diese nicht in Bezug auf alle Taten, sondern nur in Bezug auf die als am schlimmsten eingestufte Tat erfasst wurde; 2011 wurde die Anzeigequote dagegen für die einzelnen Delikte erhoben. Auf die Darstellung der Anzeigequote für 2011 wurde dennoch nicht verzichtet, um aufzuzeigen, dass die Anzeigebereitschaft für dieses Delikt mit 13.4\% besonders niedrig ausfällt im Vergleich zur Körperverletzung und zur Verge- 
waltigung. Bei den Prävalenzraten zeigt sich, dass der sexuelle Kindesmissbrauch rückläufig ist, und zwar von 6.5 auf $4.4 \%$ (vgl. Stadler et al., 2012). Die Raten beziehen sich auf Missbrauchserlebnisse bis zum 16. Lebensjahr. Da der Großteil der Befragten deutlich älter ist, beschreiben diese Zahlen die weiter zurückliegende Vergangenheit. Würde die aktuelle Kinder- und Jugendgeneration befragt werden, lägen die Prävalenzraten sicherlich noch einmal niedriger. Für Frauen hat sich die Prävalenzrate weniger stark verringert als für Männer, weshalb in diesem Deliktsbereich letztlich festzustellen ist, dass sich das Verhältnis der Raten vergrößert.

Tabelle 1. Entwicklung von Gewaltopferprävalenzraten und Anzeigequoten nach Geschlecht und Jahr (in \%).

\begin{tabular}{|c|c|c|c|c|c|c|c|c|}
\hline & & \multicolumn{4}{|c|}{ 5-Jahres-Prävalenzrate } & \multicolumn{3}{|c|}{ Anzeigequote } \\
\hline & & insg. & männl. & weibl. & $\begin{array}{c}\text { Ver- } \\
\text { hält- } \\
\text { nis } \\
\end{array}$ & insg. & männl. & weibl. \\
\hline \multicolumn{9}{|c|}{ Repräsentativbefragung } \\
\hline \multirow{2}{*}{ Raub } & 2004 & 2.2 & 2.3 & 2.0 & 1.13 & & & \\
\hline & 2006 & 2.0 & 2.5 & 1.6 & 1.56 & & & \\
\hline \multirow{3}{*}{$\begin{array}{l}\text { Körperver- } \\
\text { letzung }\end{array}$} & 2004 & 4.6 & 6.9 & 2.7 & 2.56 & & & \\
\hline & 2006 & 4.1 & 6.2 & 1.9 & 3.23 & & & \\
\hline & 2010 & 3.0 & 4.4 & 1.7 & 2.61 & & & \\
\hline \multicolumn{9}{|c|}{ Victimsurvey } \\
\hline \multirow{3}{*}{$\begin{array}{l}\text { Körperver- } \\
\text { letzung }\end{array}$} & $1992 *$ & 6.8 & 10.0 & 6.6 & 2.77 & 24.2 & 23.6 & 25.9 \\
\hline & $1992 *$ & 7.9 & 11.6 & 4.3 & 2.74 & & & \\
\hline & 2011 & 12.5 & 17.6 & 7.3 & 2.40 & 30.3 & $\begin{array}{l}31.4 \\
31.4\end{array}$ & 27.7 \\
\hline \multirow{3}{*}{$\begin{array}{l}\text { Vergewal- } \\
\text { tigung }\end{array}$} & $1992 *$ & 1.7 & 0.2 & 3.1 & 0.08 & 22.2 & 0.0 & 23.5 \\
\hline & $1992 *$ & 2.7 & 0.5 & 4.8 & 0.10 & & & \\
\hline & 2011 & 1.2 & 0.3 & 2.2 & 0.14 & 16.9 & 0.0 & 19.2 \\
\hline \multirow{2}{*}{$\begin{array}{l}\text { Sexueller } \\
\text { Kindes- } \\
\text { missbrauch }\end{array}$} & 1992 & 6.5 & 3.2 & 9.6 & 0.33 & & & \\
\hline & 2011 & 4.4 & 1.5 & 7.4 & 0.21 & 13.4 & 11.4 & 14.0 \\
\hline
\end{tabular}

Anmerkung. * vgl. für Erläuterung der zweifachen Darstellung eines Jahres Ausführungen im Text, Repräsentativbefragung sowie Victimsurvey 2011: gewichtete Daten. 


\subsection{Spezifische Bevölkerungsgruppen}

\section{Polizeibeamte}

Das Ausmaß der Gewalt gegen Polizeibeamte lässt sich teilweise auf Basis der Polizeilichen Kriminalstatistik bestimmen, da entsprechende Übergriffe, sofern sie angezeigt werden, erfasst werden. Allerdings ist die Erfassung mit verschiedenen Problemen behaftet (vgl. Ellrich, 2012). Unter dem Schlüssel „Widerstand gegen die Staatsgewalt“ sind bspw. nicht ausschließlich Polizeibeamte als Opfer zu finden, sondern auch andere Beamte (z. B. Vollzugsbeamte). Zudem werden in diesem Schlüssel eher leichtere Widerstandshandlungen (z. B. sich sperren, Anweisungen nicht Folge leisten) erfasst; physische Gewaltübergriffe werden unter den verschiedenen Schlüsseln der Körperverletzung registriert, wobei hier Polizeibeamte in der Vergangenheit nicht identifizierbar waren. Zwar hat es in jüngster Vergangenheit Veränderungen in den Erfassungsmodalitäten gegeben, Aussagen zu Entwicklungstrends lassen sich damit aber noch nicht treffen. Hierzu muss weiterhin auf den Schlüssel zu den Widerstandshandlungen zurückgegriffen werden. Dieser belegt zwischen 1993 und 2008 einen kontinuierlichen Anstieg der Zahlen (vgl. Ellrich, Baier \& Pfeiffer, 2012): Während im Jahr 1993 nur 18,293 Widerstandshandlungen registriert wurden, waren es 2008 28,272. Danach setzt allerdings wieder ein Rückgang ein; 2011 werden noch 22,839 Handlungen ausgewiesen.

Der Anstieg der registrierten Widerstandshandlungen war für zehn Innenministerien der Länder im Jahr 2009 Anlass dafür, das KFN mit einer Dunkelfelduntersuchung zur Gewalt gegen Polizeibeamte zu beauftragen (vgl. Ellrich et al., 2012). An einer Online-Befragung haben sich aus diesen Bundesländern 20,938 Beamte beteiligt (Rücklaufquote 25.1\%). Die Gewaltopfererfahrungen wurden in zweifacher Hinsicht erhoben:

- Zum einen sollten die Befragten angeben, wie häufig sie in den zurückliegenden zwölf Monaten verschiedene Übergriffe erlebt haben.

- Zum anderen wurde ein Schwerpunkt auf die Erfassung solcher Gewaltübergriffe gelegt, die zu mindestens eintägiger Dienstunfähigkeit geführt haben. In der Rückschau auf die Jahre 2005 bis 2009 sollten alle Übergriffe mit nachfolgender Dienstunfähigkeit berichtet werden, so dass es möglich ist, für diesen Zeitraum eine Entwicklung sichtbar zu machen (und mit den Befunden der Polizeilichen Kriminalstatistik zu vergleichen). 
Die Ergebnisse aus Tabelle 2 belegen, dass unabhängig davon, welcher Opferindikator betrachtet wird, weibliche Beamte signifikant seltener viktimisiert werden als männliche Beamte. Die auf die letzten zwölf Monate bezogenen Viktimisierungen stellen z. T. Indexvariablen dar, d. h. hier wurden Angaben zu mehreren Items zusammengefasst. Dies geschah derart, dass die höchste Häufigkeit gewertet wurde. Davon, in den letzten zwölf Monaten festgehalten, angepackt, geschubst oder gestoßen worden zu sein, berichten $41.8 \%$ der weiblichen, aber $49.5 \%$ der männlichen Beamten (,festhalten/schubsen"). Am seltensten wurde angegeben, das Drohen bzw. den Einsatz von Waffen (inkl. Schusswaffen) erlebt zu haben; auch hier finden sich signifikante Geschlechterunterschiede derart, dass weibliche Beamte seltener viktimisiert wurden als männliche Beamte (13.2 zu 18.4\%).

Tabelle 2. Gewaltviktimisierung von Polizeibeamten nach Geschlecht (in \%, fett: Unterschiede signifikant bei $p<.05$ ).

\begin{tabular}{|c|c|c|c|}
\hline & & weibl. & männl. \\
\hline \multirow{4}{*}{ letzte 12 Monate } & festhalten/schubsen & 41.8 & 49.5 \\
\hline & schlagen/treten & 23.1 & 27.3 \\
\hline & mit Gegenständen bewerfen & 21.6 & 25.4 \\
\hline & Drohen bzw. Einsetzen Waffe & 13.2 & 18.4 \\
\hline \multirow{6}{*}{$\begin{array}{l}\text { Übergriff mit Dienstun- } \\
\text { fähigkeit }\end{array}$} & 2005 & 1.3 & 2.3 \\
\hline & 2006 & 1.2 & 2.4 \\
\hline & 2007 & 1.5 & 2.8 \\
\hline & 2008 & 1.4 & 3.2 \\
\hline & 2009 & 2.5 & 3.7 \\
\hline & insgesamt & 6.6 & 11.2 \\
\hline
\end{tabular}

Mindestens einen Übergriff mit mindestens eintägiger Dienstunfähigkeit in den Jahren 2005 bis 2009 berichten 6.6\% der weiblichen und $11.2 \%$ der männlichen Beamten. Für jedes Jahr kann dabei ein signifikanter Geschlechterunterschied festgestellt werden. Auch in der Dunkelfeldbefragung zeichnet sich ein Anstieg der Gewaltübergriffe auf Polizeibeamte ab, was die Auswertungen zu den Widerstandsdelikten der Polizeilichen Kriminalstatistik stützt. Gleichwohl geschieht der Anstieg in unterschiedlichem Tempo: Bei Frauen bleibt der Anteil zwischen 2005 und 2008 weitestgehend konstant; erst die Rate für das Jahr 2009 liegt mit 
$2.5 \%$ deutlich höher als die Raten vorher. Bei Männern ist hingegen ein kontinuierlicher Anstieg über die Jahre festzustellen. Letztlich kann daher keine klare Aussage darüber getroffen werden, wie sich der Geschlechterunterschied über die Jahre entwickelt hat. Von einer deutlichen Annäherungsbewegung ist aber nicht auszugehen.

Eine zusätzliche Auswertung bestätigt, dass Frauen seltener angegriffen werden und dass die Gegenwart von Frauen im Einsatzteam einen entsprechenden Ausstrahlungseffekt hat. Im Rahmen der Befragung wurde nach den letzten Einsätzen bei häuslicher Gewalt gefragt (Ellrich et al., 2012, S. 163ff). Bezogen auf jene Einsätze, bei denen zwei Beamte vor Ort waren, zeigt sich, dass es mit einer Beamtin im Einsatzteam in 5.4\% der Fälle zur Verletzung mindestens eines Beamten kam; in Situationen mit zwei männlichen Beamten beträgt die Verletzungsrate hingegen 6.0\%. Dabei ist für männliche wie für weibliche Beamte das Risiko des Verletztwerdens geringer, wenn sie in gemischtgeschlechtlichen Teams einen solchen Einsatz haben. „Wenn ein männlicher Beamter zusammen mit einem anderen männlichen Beamten in einem Zweierteam zu einem Einsatz im Rahmen häuslicher Gewalt gerufen wird, dann ist sein Risiko, verletzt zu werden, $4.5 \%$ [...] Wenn ein männlicher Beamter mit einem weiblichen Beamten zusammen in den Einsatz geht, dann liegt das Risiko nur bei 3.6\%"(Ellrich et al., 2012, S. 179).

Dass sich die Geschlechterunterschiede in den Viktimisierungsraten von Polizeibeamten so deutlich zeigen, überrascht vor dem Hintergrund, dass Beamtinnen wie Beamte in vergleichbarem Maße potenziell gefährlichen Situationen ausgesetzt sein sollten. Als Erklärung für den Geschlechterunterschied werden verschiedene Thesen geäußert (Ellrich, 2012, S. 13). Möglicherweise haben die überwiegend männlichen Täter eine größere Hemmung, Frauen anzugreifen. Eventuell wird den Frauen während der Einsätze auch eine andere Funktion zugewiesen. Sie könnten bspw. häufiger die Sicherung übernehmen und Aufgabe des männlichen Kollegen ist es, sich als erster in die Gefahrensituation zu begeben. Zudem besteht die Annahme, dass Frauen aufgrund ihrer Sozialisation kommunikativer und sozial verträglicher agieren und gefährliche Situationen besser verbal deeskalieren können. Empirisch getestet wurden diese verschiedenen Erklärungsansätze bislang nicht. Weitere Untersuchungen zu diesem Thema erscheinen daher notwendig, insofern sich für andere Beamtenmerkmale wie das Alter oder die körperliche Statur keine systematischen Zusammenhänge mit dem Opferrisiko zeigen. Gleichwohl ist an dieser Stelle ebenfalls zu betonen, dass das Beamtengeschlecht in seinem Stellenwert für die Erklärung von Opferschaften hinter andere Variablen zurücktritt. So stellen 
bspw. die Alkoholisierung einer Person oder der Drogenkonsum wichtigere Erklärungsvariablen dar (Ellrich et al., 2012, S. 177).

\section{Strafgefangene}

In Bezug auf Strafgefangene existiert keine der Polizeilichen Kriminalstatistik vergleichbare Hellfeldstatistik, die über die Häufigkeit der Opferwerdung Auskunft geben könnte. Nur über die Analyse von Personalakten in den Gefängnissen lässt sich ein Bild zur Hellfeld-Kriminalität nachzeichnen. Dies hat u. a. Heinrich (2002) für den hessischen, Wirth (2007) für den nordrhein-westfälischen Justizvollzug getan. Daneben finden sich zur Viktimisierung von Strafgefangenen Dunkelfeldbefragungen (u. a. Ernst, 2008). Eine aktuelle Studie wurde durch das KFN durchgeführt, das in den Jahren 2011 und 2012 in 48 Strafvollzugsanstalten in fünf Bundesländern in vergleichbarer Weise Inhaftierte befragt hat. Bei den Bundesländern handelt es sich um Brandenburg, Bremen, Niedersachsen, Sachsen und Thüringen (vgl. Baier \& Bergmann, 2013). An der Befragung haben 5,983 Gefangene teilgenommen (Rücklaufquote: 50.3\%). Im Erwachsenenvollzug wurden dabei 4,436 Männer und 460 Frauen erreicht; im Jugendstrafvollzug 1,087 Personen.

Im Fragebogen wurden u. a. verschiedene Opfererfahrungen erfasst. Einzuschätzen war, wie häufig folgende Verhaltensweisen in den letzten vier Wochen erlebt worden sind:

- Sexuelle Gewalt: Ich musste Mitgefangene mit dem Mund befriedigen; Ich wurde zum Geschlechtsverkehr/Analverkehr gezwungen.

- Physische Gewalt: Ich wurde mit Absicht gestoßen; ich wurde mit der Hand/Faust geschlagen oder getreten; ich wurde gequält/gefoltert; ich wurde mit einem Gegenstand geschlagen.

- Erpressung: Ich musste Mitgefangenen Einkauf bezahlen; ich musste Familie/Freunde bitten, Mitgefangenen Geld zu schicken; ich wurde angewiesen, Mitgefangenen Geld zu schicken, wenn ich rauskomme; ich musste etwas von meinem Einkauf abgeben; ich wurde gezwungen, Mitgefangenen meine Telefonkarte/meinen PIN-Code zu geben.

Die Einzelitems wurden über den Maximalwertbefehl zu den genannten Indizes zusammengefasst. Die höchste Häufigkeit zu einer Verhaltensweise bestimmt damit den Indexwert. Wenn bspw. ein Befragter selten gezwungen wurde, Mitgefangene mit dem Mund zu befriedigen, zugleich 
aber nie zum Geschlechtsverkehr gezwungen wurde, erhält der Befragte den Wert „selten“ auf dem Index ,sexuelle Gewalt“. Allerdings werden im Folgenden nur Prävalenzraten berichtet: Es standen zwar als Antwortvorgaben die Möglichkeiten „nie“, „selten“, „manchmal“ und „häufig“ zur Verfügung. Da aber insbesondere die Kategorie „häufig“ nur sehr selten angekreuzt wurde, erscheint es nicht sinnvoll, differenziertere Auswertungen vorzunehmen.

Entsprechend der Ergebnisse kann gefolgert werden, dass Gewalterlebnisse im Strafvollzug keine Seltenheit darstellen (Baier \& Bergmann, 2013): 16.8\% der Befragten des Männervollzugs geben an, in den letzten vier Wochen physische Gewalt erfahren zu haben, bei den Frauen beträgt die Quote 11.4\%. Der Unterschied zwischen den Geschlechtern wird als signifikant ausgewiesen. Für die anderen Gewaltformen gilt dies gleichwohl nicht: Bei den Erpressungen betragen die Prävalenzraten 11.4\% (Männer) und $12.7 \%$ (Frauen); hier ergibt sich also eine höhere Belastung für Frauen, wobei der Unterschied nicht signifikant ist. Auch bei der sexuellen Gewalt findet sich kein signifikanter Unterschied, wobei die Männer zugleich höhere Raten aufweisen als die Frauen (2.1 zu 1.1\%). Werden die Täterschaften einbezogen, die ebenfalls im Rahmen der Befragung erhoben wurden und die komplementär zu den Opfererfahrungen erfragt wurden, ergeben sich für keine der drei Gewaltformen signifikante Geschlechtsunterschiede. Im Strafvollzug, d. h. unter eine Gruppe kriminell höchst auffälliger Personen, sind Geschlechterunterschiede im Gewaltverhalten (aus Opfer- wie Täterperspektive) mithin kaum mehr auszumachen.

Exkurs: Verbale und relationale Aggression im Jugendalter

Bisher standen Gewaltverhaltensweisen im Vordergrund, die als straffällig einzustufen sind. In der deutschlandweit repräsentativen Schülerbefragung 2007/2008 wurde zusätzlich auch nach dem Begehen von Taten gefragt, die zwar als aggressiv zu bezeichnen sind, die aber nicht gegen normative Vorgaben verstoßen. In Bezug auf den Kontext Schule sollten die Befragten angeben, wie häufig sie im letzten Schulhalbjahr folgende Verhaltensweisen ausgeführt haben:

- Verbale Aggression: einen anderen Schüler gehänselt oder hässliche Dinge über ihn gesagt; 
- Relationale Aggression: Freunde aufgefordert, damit aufzuhören, mit anderen Schülern etwas zu unternehmen; andere Schüler wie Luft behandelt und absichtlich nicht mehr beachtet;

- Lehrermobbing: eine Lehrkraft vor anderen Schülern lächerlich gemacht; eine Lehrkraft richtig gemein behandelt.

Als Antwortvorgaben standen Optionen von „, 1 - nie“ bis , 6 - mehrmals pro Woche" zur Verfügung. Nachfolgend wird zwischen jenen Schülern unterschieden, die die Verhaltensweisen sporadisch zeigen (bis zu sechs Mal in letzten Schulhalbjahr) und Schülern, die noch häufiger das Verhalten ausgeführt haben. Bei den Indizes, die mit zwei Items gemessen wurden, geht jeweils das Item mit der höchsten Häufigkeit in die Auswertungen ein.

Abbildung 4 belegt, dass es im Bereich der verbalen Aggression wie im Bereich des Lehrermobbings die bekannten Geschlechterunterschiede gibt, nach denen Jungen häufiger als Täter in Erscheinung treten. Die Unterschiede sind aber gerade bei den Anteilen sporadischer Täter deutlich geringer als bei den oben betrachteten physischen Gewalttaten. Mindestens mehrfach monatlich werden verbale Angriffe bzw. Angriffe auf Lehrkräfte allerdings deutlich häufiger von Jungen als von Mädchen berichtet.

Die Betrachtung relationaler Verhaltensweisen zeigt einen umgekehrten Geschlechtereffekt: Diese aggressiven Verhaltensweisen werden häufiger von Mädchen als von Jungen ausgeführt. Insgesamt 42.6\% der Mädchen haben sich relational aggressiv verhalten, bei den Jungen waren es nur $36.5 \%$. Die Unterschiede finden sich im Übrigen in allen Schulformen (vgl. Baier, 2011c). Je indirekter aggressives Verhalten ist und je weniger zumindest in physischer Hinsicht ein Verhalten als schädigend einzustufen ist, umso häufiger treten Mädchen als Täter in Erscheinung. 


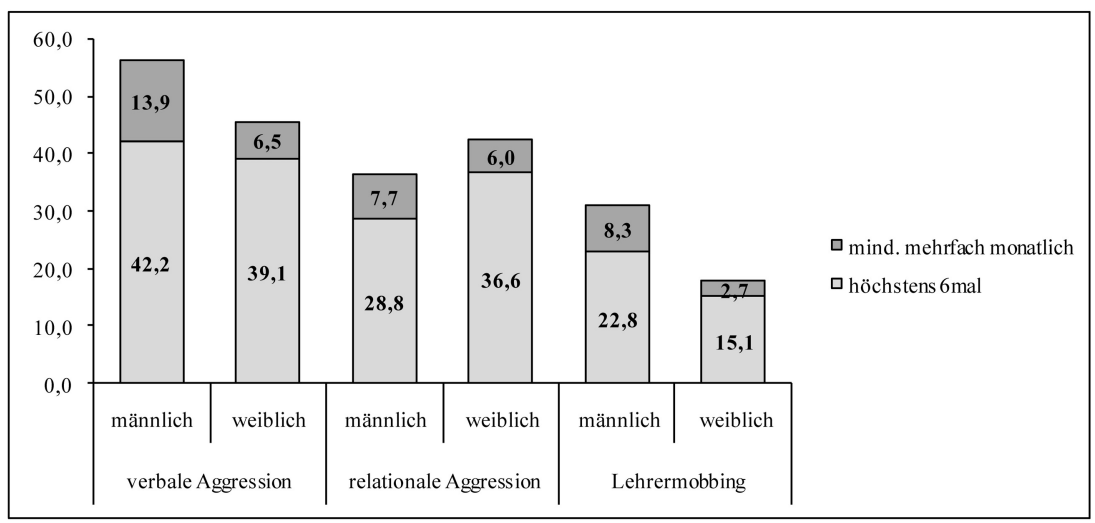

Abbildung 4. Häufigkeit aggressiven Verhaltens in der Schule im letzten Schulhalbjahr nach Geschlecht (in \%).

Bezüglich der Entwicklung dieser Verhaltensweisen liegen bislang kaum empirische Erkenntnisse vor. Nur in drei Gebieten konnten bislang in wiederholt durchgeführten Schülerbefragungen Befunde zur verbalen Aggression erarbeitet werden. So berichtet Baier (2008, S. 29ff), dass in München der Anteil verbal aggressiver Schüler im Vergleich der Jahre 2000 und 2005 nahezu unverändert geblieben ist, in Hannover hat er dagegen signifikant abgenommen. Dieses Ergebnis wird auch für eine in den Jahren 2005 und 2010 durchgeführte Wiederholungsbefragung in einem niedersächsischen Landkreis (Soltau-Fallingbostel) erzielt (Baier, 2011a, S. 38ff). Eine nach dem Geschlecht differenzierte Auswertung ergibt dabei, dass in Hannover die Raten bei männlichen Jugendlichen, im Landkreis Soltau-Fallingbostel die Raten bei weiblichen Jugendlichen stärker gefallen sind. Die Befunde lassen damit keinen eindeutigen Schluss bzgl. möglicher geschlechtsspezifischer Entwicklungstrends zu. Einzig die Folgerung, dass auch andere als physische Aggressionen tendenziell rückläufig sind, kann auf Basis der wenigen Befunde abgeleitet werden. 


\section{Eigentumskriminalität}

\subsection{Jugendliche als Täter}

Polizeiliche Kriminalstatistik

Die zwei im Jugendalter verbreitetsten Eigentumsdelikte sind der Ladendiebstahl und die Sachbeschädigung. Das Jahr 2011 betrachtet, wurden 1.6\% (51,374 Personen) aller in Deutschland lebenden Jugendlichen wegen eines einfachen Ladendiebstahls polizeilich registriert, bei der Sachbeschädigung waren es 1.0\% (33,020 Personen). Der Anteil wegen eines Ladendiebstahls polizeilich als tatverdächtig registrierten Jungen lag dabei im Jahr 2011 bei $1.5 \%$, bei den Mädchen bei 1.7\%. Der einfache Ladendiebstahl ist damit das einzige Delikt im Jugendalter, bei dem zumindest in der Hellfeldstatistik mehr Mädchen als Jungen als Täter in Erscheinung treten. Bei der Sachbeschädigung ist hingegen der klassische gender gap mit höheren Belastungen bei den Jungen festzustellen: 1.8\% aller männlichen Jugendlichen und $0.2 \%$ aller weiblichen Jugendlichen wurden im Jahr 2011 wegen einer Sachbeschädigung als Tatverdächtige polizeilich registriert.

Welche Entwicklung es in beiden Deliktsbereichen seit 1997 gegeben hat, ist in Abbildung 5 dargestellt. Die Tatverdächtigenbelastungszahl für Ladendiebstahl ist demnach deutlich gesunken; bei der Sachbeschädigung ergeben sich zumindest in den letzten Jahren leicht sinkende Zahlen. Bei beiden Delikten kommt es im Zeitverlauf zu einer Annäherung der Geschlechter. Besonders deutlich ist diese Entwicklung beim Ladendiebstahl: Im Jahr 1997 wurden männliche Jugendliche noch 1.3-mal häufiger als weibliche Jugendliche als Tatverdächtige dieses Delikts registriert; im Jahr 2011 hat sich dies umgekehrt, da nun Mädchen fast 1.2-mal häufiger als Jungen registriert werden. Bei der Sachbeschädigung ist das Verhältnis von eins zu zehn auf eins zu acht gefallen; deutliche Geschlechterunterschiede bleiben hier zugleich weiterhin bestehen. 


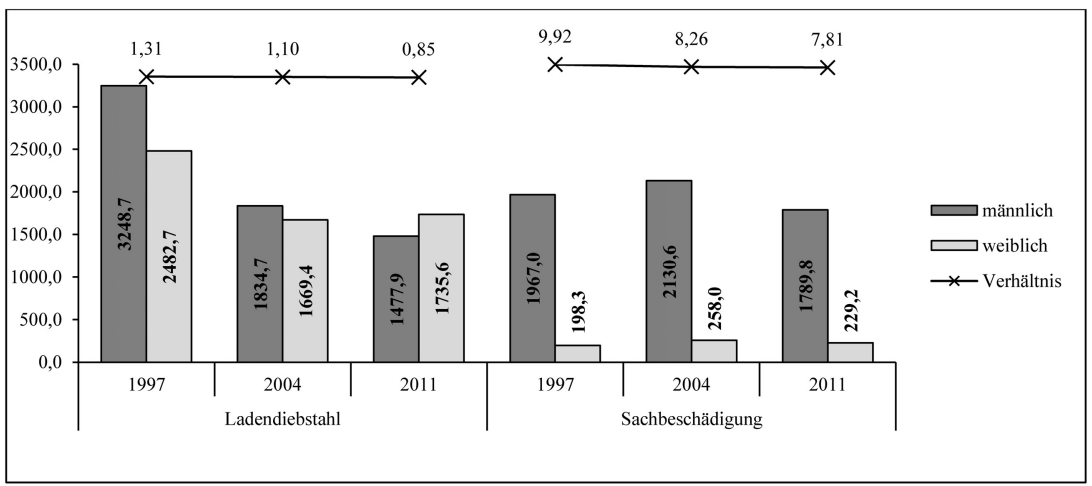

Abbildung 5. Tatverdächtigenbelastungszahl Jugendlicher für verschiedene Eigentumsdelikte nach Geschlecht und Jahr.

\section{Dunkelfeldbefragung}

Auch bezüglich der Delikte Ladendiebstahl und Sachbeschädigung gilt, dass die Täterrate im Dunkelfeld die des Hellfelds deutlich übersteigt, nur ein Teil aller Taten wird mithin entdeckt und angezeigt. In der Schülerbefragung 2007/2008 gaben 13.3\% der Befragten an, in den zurückliegenden zwölf Monaten mindestens einen Ladendiebstahl verübt zu haben, 14.6\% haben mindestens eine Sachbeschädigung ausgeführt. Beim Ladendiebstahl liegt die Dunkelfeldrate also in etwa achtmal höher als die Hellfeldrate, bei der Sachbeschädigung 14-mal. Ob die Anzeigequote entsprechend niedriger liegt, lässt sich mit dieser Schülerbefragung nicht beantworten, weil die Anzeigequote nicht erfragt worden ist. In einer Schülerbefragung in Hannover im Jahr 2006 wurden die Entdeckungsquoten ermittelt (Rabold, Baier \& Pfeiffer, 2008, S. 42). Diese bestätigen, dass Ladendiebstähle häufiger polizeilich registriert werden als Sachbeschädigungen: Beim Ladendiebstahl beträgt die Entdeckungsrate $8.2 \%$, bei der Sachbeschädigung 3.8\%.

Der Vergleich der Prävalenzraten nach dem Geschlecht bestätigt tendenziell die Hellfeldbefunde: Mädchen geben fast genauso häufig an wie Jungen, in den letzten zwölf Monaten mindestens einen Ladendiebstahl ausgeführt zu haben (12.4 zu 14.2\%), bei der Sachbeschädigung liegen ihre Täterraten dagegen deutlich niedriger (7.1 zu 21.9\%).

Wird die Entwicklung der Prävalenzraten für beide Delikte für die Jahren 1998 und 2005/2006 in den Städten München, Stuttgart, Hannover und Schwäbisch Gmünd betrachtet, so zeigt sich für den Ladendiebstahl fol- 
gendes Bild: Der Anteil an Tätern ist von 32.7 auf $16.9 \%$ deutlich zurückgegangen (Baier, 2008, S. 32), was die Befunde der Kriminalstatistik stützt. Ebenfalls bestätigt wird, dass für männliche Jugendliche ein stärkerer Rückgang zu verzeichnen ist als für weibliche Jugendliche, was zur Folge hat, dass Jungen nicht mehr 1.2-mal, sondern nur noch 1.1-mal häufiger als Mädchen Täter von Ladendiebstählen sind (Baier, 2011c, S. 360).

Bei den Sachbeschädigungen ergeben sich der Kriminalstatistik widersprechende Befunde, in zweifacher Hinsicht. Erstens geht der Anteil an Tätern über die Jahre signifikant zurück, von 16.3 auf $13.2 \%$. Zweitens gleichen sich die weiblichen Jugendlichen den männlichen Jugendlichen nicht allmählich an, sondern die Schere wird größer. Jungen haben im Jahr 1998 2.2-mal häufiger als Mädchen Sachbeschädigungen ausgeführt, 2005/2006 haben sie dies 2.4-mal häufiger getan. Auch hier liegt, wie im Bereich des Gewaltverhaltens, die These nahe, dass einerseits die Anzeigequote gestiegen ist, andererseits vor allem weibliche Täter verstärkt im Hellfeld sichtbar werden. Prüfen lassen sich diese Annahmen mit den vorhandenen Daten jedoch nicht, da die Anzeige- bzw. Entdeckungswahrscheinlichkeit in Bezug auf Eigentumsdelikte in diesen Befragungen nicht erfragt wurde.

\subsection{Opferschaften in der Erwachsenenbevölkerung}

\section{Polizeiliche Kriminalstatistik}

Entgegen den Gewaltdelikten erlaubt die Polizeiliche Kriminalstatistik nicht, die Opfergefährdungszahl für Eigentumsdelikte $\mathrm{zu}$ bestimmen. Auch eine Differenzierung zwischen den Geschlechtern ist in der Kriminalstatistik mit Blick auf die Eigentumsdelikte nicht möglich. Um dennoch einige ausgewählte Entwicklungstrends der Eigentumskriminalität berichten zu können, wird an dieser Stelle auf die registrierten Fälle Bezug genommen. Werden die Fälle betrachtet, ist eine Einschränkung der Analysen auf Erwachsene bzw. eine Darstellung für die Geschlechter nicht möglich, da die Fälle unabhängig vom Täter oder vom Opfer (und deren Eigenschaften) sind. Es stehen hier also allgemeine Trends im Bereich der Eigentumskriminalität im Vordergrund.

Für die nachstehend ausgewählten Delikte kann auf Basis der Polizeilichen Kriminalstatistik Folgendes gesagt werden: 
- Der Diebstahl insgesamt ist in Deutschland deutlich rückläufig. Im Jahr 1993 wurden deutschlandweit noch 4.2 Mio. Diebstähle registriert, 2011 waren es nur mehr 2.4 Millionen. Diese Entwicklung zeigt sich auch in zwei Unterkategorien: dem einfachen Ladendiebstahl und dem Wohnungseinbruchsdiebstahl. Der Ladendiebstahl hat von ca. 660,000 auf 370,000 Fälle abgenommen, der Wohnungseinbruchsdiebstahl von ca. 230,000 auf 130,000 Fälle. Beim Wohnungseinbruchsdiebstahl ist zugleich auffällig, dass die wenigsten Diebstähle bereits im Jahr 2006 mit etwa 100,000 Einbruchsdiebstählen registriert wurden, seit dem steigt der Wohnungseinbruch kontinuierlich an.

- Sachbeschädigungen nehmen über die Jahre hinweg leicht zu: 1993 gab es ca. 580,000 Sachbeschädigungen in Deutschland, im Jahr 2011 ca. 690,000. Die höchste Zahl an Sachbeschädigungen wurde allerdings bereits im Jahr 2008 mit ca. 800,000 Fällen registriert.

- Ein weiteres in Deutschland recht weit verbreitetes Eigentumsdelikt ist der Betrug. Hier ergeben sich mit die deutlichsten Kriminalitätsanstiege: Im Jahr 1993 wurden ca. 530,000 Betrugsfälle polizeilich registriert, 2011 waren es ca. 930,000. Die höchste Anzahl an Delikten wurde ein Jahr zuvor mit etwa 970,000 Fällen registriert.

Die Betrachtung der zahlenmäßig wichtigsten Eigentumsdelikte ergibt damit ein widersprüchliches Bild. Der Diebstahl geht zurück, während Sachbeschädigungen und Betrugsdelikte ansteigen. Die Erklärung für diese Trends kann daher nicht auf einen einzelnen Faktor setzen (vgl. Baier et al., 2011, S. 13ff). Der Rückgang der Diebstahlsdelikte dürfte damit in Zusammenhang stehen, dass sich Sicherheitstechniken immer weiter durchgesetzt haben, die den Diebstahl erschweren. Der Anstieg der Betrugsdelikte könnte in erster Linie mit den neuen Betrugsmöglichkeiten im Internet erklärt werden. Für den Anstieg der Sachbeschädigung könnten eine gestiegene Sensibilisierung und damit eine erhöhte Anzeigebereitschaft verantwortlich sein.

\section{Dunkelfeldbefragung}

Die deutschlandweiten Repräsentativbefragungen 2004, 2006 und 2010 sowie der Victimsurvey 1992 und 2011 erlauben nur zu sehr wenigen Eigentumsdelikten Auswertungen zum Dunkelfeld. In der Repräsentativbefragung wurde zum Einen nach dem Erleben eines Diebstahls (Gegen- 
stände, Geld oder andere Zahlungsmittel oder sonstige wichtige Dokumente gestohlen) gefragt. Die Fünf-Jahres-Prävalenzen sind dabei rückläufig, was die Befunde des Hellfelds stützt (Tabelle 3). Für Frauen und Männer erfolgt der Rückgang im vergleichbaren Ausmaß, so dass weiterhin Männer etwa 1.2-mal häufiger von Diebstählen betroffen sind als Frauen.

Zum anderen wurde in der Repräsentativbefragung nach dem Erleben eines Wohnungseinbruchsdiebstahls gefragt. Im Vergleich der Jahre 2004 und 2006 sinkt die Prävalenzrate von 3.1 auf $2.4 \%$, bei Männern wie bei Frauen. Auch damit werden Erkenntnisse des Hellfelds gestützt, in dem zumindest in diesem Zeitraum ein Rückgang des Wohnungseinbruchsdiebstahls ausgewiesen wird.

Auch der Victimsurvey erlaubt die Untersuchung des Erlebens von Wohnungseinbrüchen, und zwar über einen deutlich längeren Zeitraum (für die 16- bis 40-jährige Wohnbevölkerung). Dabei wird eine rückläufige Entwicklung sichtbar. Dies stützt die Kriminalstatistik, in der in einem solch langen Zeitvergleich ebenso ein Rückgang sichtbar wird. Erst seit dem Jahr 2007 steigt der Wohnungseinbruch in der Kriminalstatistik an, jedoch nicht auf das hohe Niveau von Anfang der 1990-er Jahre. Interessant ist, dass sich für Männer ein geringerer Rückgang der Einbruchsprävalenz zeigt als für Frauen, was zur Folge hat, dass das Verhältnis der Prävalenzraten ansteigt. Plausibel ist eine solche Entwicklung nicht, da nicht anzunehmen ist, dass Einbrecher eher in Wohnungen männlicher als in Wohnungen weiblicher Personen einbrechen. Möglicherweise drücken sich in dem Unterschied der Prävalenzraten von Männern und Frauen Wahrnehmungsunterschiede bspw. bezüglich eines versuchten Einbruchs oder eines Einbruchs in Räume, die nicht unmittelbar zur Wohnung gehören (z. B. Geschäftsräume, Keller) aus. Was das Anzeigeverhalten anbelangt, sind Geschlechterunterschiede vernachlässigbar. Grundsätzlich werden Einbruchsdiebstähle sehr häufig angezeigt; dies war im Jahr 1992 nicht anders als im Jahr 2011. 
Tabelle 3. Entwicklung von Eigentumsdeliktopferraten und Anzeigequoten nach Geschlecht und Jahr (in \%).

\begin{tabular}{|c|c|c|c|c|c|c|c|c|c|}
\hline & & & \multicolumn{4}{|c|}{ 5-Jahres-Prävalenzrate } & \multicolumn{3}{|c|}{ Anzeigequote } \\
\hline & & & $\mathrm{i}$ & $\mathrm{m}$ & $\mathrm{w}$ & $\begin{array}{l}\text { Ver- } \\
\text { hält- } \\
\text { nis } \\
\end{array}$ & $\mathrm{i}$ & $\mathrm{m}$ & w \\
\hline \multirow{6}{*}{$\begin{array}{l}\text { Reprä- } \\
\text { sen- } \\
\text { tativbe- } \\
\text { fragung }\end{array}$} & \multirow{3}{*}{ Diebstahl } & 2004 & 12,3 & 13,6 & 11,2 & 1,22 & & & \\
\hline & & 2006 & 12,0 & 14,7 & 9,5 & 1,55 & & & \\
\hline & & 2010 & 9,6 & 10,8 & 8,5 & 1,27 & & & \\
\hline & \multirow{3}{*}{$\begin{array}{l}\text { Wohnungs- } \\
\text { einbruchs- } \\
\text { diebstahl }\end{array}$} & 2004 & 3,1 & 3,3 & 2,9 & 1,13 & & & \\
\hline & & 2006 & 2,4 & 2,6 & 2,3 & 1,16 & & & \\
\hline & & 1992 & 3,2 & 3,3 & 3,2 & 1,06 & & & \\
\hline \multirow{2}{*}{$\begin{array}{l}\text { Victim- } \\
\text { survey }\end{array}$} & \multirow{2}{*}{$\begin{array}{l}\text { Wohnungs- } \\
\text { einbruchs- } \\
\text { diebstahl }\end{array}$} & 2011 & 2,1 & 2,5 & 1,7 & 1,44 & 78,6 & 76,5 & 81,8 \\
\hline & & 2004 & 12,3 & 13,6 & 11,2 & 1,22 & 81,2 & 81,7 & 81,3 \\
\hline
\end{tabular}

Anmerkung. $\mathrm{i}=$ insgesamt. $\mathrm{m}=$ männlich. $\mathrm{w}=$ weiblich.

4. Kriminalitätswahrnehmung, Kriminalitätsfurcht und Strafbedürfnisse unter Erwachsenen

Um Geschlechtsunterschiede in kriminalitätsbezogenen Wahrnehmungen und Einstellungen zu untersuchen, wird auf die deutschlandweit repräsentativen Befragungen der Jahre 2004, 2006 und 2010 zurückgegriffen (Baier et al., 2011). In allen Erhebungsjahren wurden folgende Bereiche untersucht:

1. Die Einschätzung, ob Straftaten in Deutschland seltener oder häufiger geworden sind: Hierbei sollte zurückblickend auf die letzten 10 Jahre angegeben werden, ob Straftaten von ,1 - sehr viel seltener" bis , ,7 - sehr viel häufiger" geworden sind. Es wurde nach verschiedenen Delikten (z. B. Körperverletzung, Wohnungseinbruch), aber auch nach ,allen Straftaten insgesamt“ gefragt. Für die Darstellung hier wird sich auf ,alle Straftaten“ beschränkt. Ausgewiesen wird der Anteil an Befragten, die mit „viel“" oder „sehr viel häufiger" geantwortet haben.

2. Die Kriminalitätsfurcht: In Anlehnung an andere Studien (u. a. Bilsky et al., 1992) wurden zwei Formen der Furcht unterschieden, die personale Kriminalitätsfurcht und das Vermeidungsverhalten. Bezüglich der personalen Furcht sollten die Befragten einschätzen, wie häufig sie die Befürchtung haben, Opfer verschiedener Straftaten zu werden; zudem sollten sie einschätzen, als wie 
wahrscheinlich sie es erachten, Opfer dieser Straftaten zu werden (u. a. Raub, Diebstahl). Bei beiden Skalen standen fünfstufige Antwortvorgaben zur Verfügung. Die personale Furcht ergibt sich aus der Multiplikation beider Angaben, wobei Werte zwischen 1 und 25 erreicht werden können. Befragte mit einem Wert von über 9 weisen eine mittlere bzw. hohe Furcht auf. Das Vermeidungsverhalten wurde über vier Aussagen wie „Ich meide bestimmte Straßen, Plätze oder Parks" (um sich vor Kriminalität zu schützen) oder "Ich vermeide es, öffentliche Verkehrsmittel zu benutzen“ (Baier et al., 2011, S. 52) erfasst, mit Antwortkategorien von „1nie“ bis ,5-immer". Befragte mit einem Mittelwert bei diesen vier Aussagen von über 3,67 weisen ein häufiges Vermeidungsverhalten auf.

3. Die Strafhärte: Die Befürwortung von strafharten Einstellungen wurde mittels vier Aussagen wie „Auf viele Straftaten sollte mit härteren Strafen reagiert werden als bisher“ oder „Harte Strafen sind notwendig, damit andere davon abgehalten werden, Straftaten zu begehen“ gemessen (Antwortvorgaben: „1 - trifft überhaupt nicht zu“ bis „6 - trifft voll und ganz zu“; Baier et al., 2011, S. 56). Mittelwerte über 4,34 indizieren damit eine hohe Strafhärte.

Das Geschlecht steht mit drei der insgesamt vier betrachteten Wahrnehmungen bzw. Einstellungen in Beziehung, wie Abbildung 6 belegt. Dies gilt auch dann, wenn weitere Faktoren wie das Alter, die Bildung oder Persönlichkeitsmerkmale in die Analyse aufgenommen werden (Baier et al., 2011, S. 150ff). Die Strafhärte ist als einziger Bereich unabhängig vom Geschlecht: Unter Frauen und Männern gibt es einen gleich hohen Anteil an Personen, die harte Strafen befürworten. Dieser Anteil ist grundsätzlich sehr hoch und hat sich über die Jahre hinweg auch nicht verändert: Etwa zwei Drittel der Befragten teilen die Ansicht, dass harte Strafen notwendig sind.

Bezüglich der anderen Einschätzungen sind zwei Befunde herauszustellen: Erstens ist im Vergleich der Jahre ein Rückgang erkennbar, zweitens weisen weibliche Befragte zu fast allen Erhebungszeitpunkten signifikant höhere Anteile auf. Besonders groß fällt der Geschlechterunterschied beim Vermeidungsverhalten aus: Bei Frauen ist der Anteil an Befragten, die häufig solches Verhalten zeigen, etwa drei Mal so hoch wie bei Männern. Gerade für die Frauen ist aber ein Rückgang des Anteils an Befragten mit häufigem Vermeidungsverhalten festzustellen. Dies gilt auch für die personale Furcht: Im Jahr 2010 unterscheiden sich die Ge- 
schlechter hinsichtlich ihrer personalen Furcht nicht mehr signifikant voneinander, 2004 und 2006 war dies noch der Fall. In diesen beiden Jahren teilte zugleich die Mehrheit der Männer und Frauen die Ansicht, dass die Anzahl an Straftaten in Deutschland stark gestiegen ist. Bei Männern und Frauen liegt dieser Anteil im Jahr 2010 deutlich niedriger als im Jahr 2004; zugleich sind Frauen weiterhin signifikant häufiger der Ansicht, die Straftaten würden stark zunehmen.

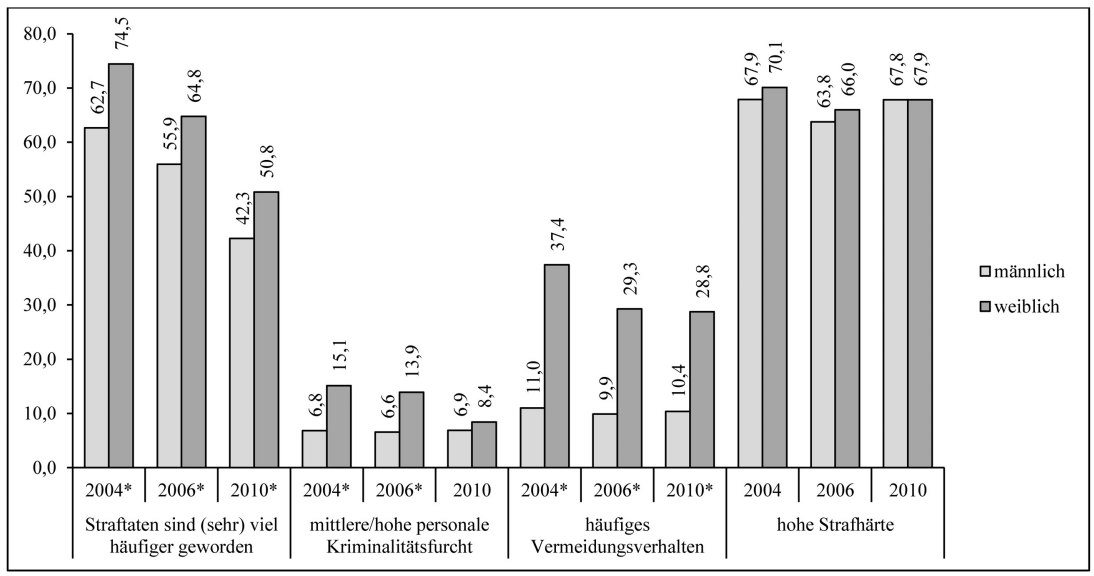

Abbildung 6. Wahrgenommene Kriminalitätsentwicklung, Kriminalitätsfurcht und Strafhärte nach Geschlecht und Jahr (in \% gewichtete Daten; * Geschlechtsunterschied signifikant bei $p<.05$ ).

Abbildung 7 geht zuletzt der Frage nach, ob sich die Geschlechtsunterschiede im Bereich der Kriminalitätseinschätzung und des Vermeidungsverhaltens altersgruppenübergreifend finden lassen. Hierzu wird auf die Befragung des Jahres 2010 zurückgegriffen. Für das Vermeidungsverhalten ist die Frage zu bejahen: Junge ebenso wie ältere Frauen ergreifen deutlich häufiger als Männer verschiedene Maßnahmen, um sich vor Übergriffen zu schützen. Mit zunehmendem Alter wird der Unterschied größer. Für beide Geschlechter gibt es zugleich einen starken Alterseffekt: Ältere Personen fürchten sich signifikant stärker als jüngere Personen vor kriminellen Übergriffen und vermeiden deshalb auch signifikant häufiger verschiedene Verhaltensweisen (vgl. Baier et al., 2011). Wird gleichzeitig das Opferrisiko betrachtet - in Abbildung 7 ist dies anhand der FünfJahres-Prävalenz für Körperverletzungen geschehen - dann erscheint die Furcht älterer Personen unbegründet: Ältere Personen berichten sehr viel 
seltener als jüngere Personen davon, Körperverletzungen in den letzten fünf Jahren erlebt zu haben. In den jüngeren Altersgruppen gibt es bezüglich der Opferprävalenz noch signifikante Geschlechterunterschiede. Bei den ab 50-Jährigen ist dies dann aber nicht mehr der Fall, d. h. zumindest im Bereich des Gewaltverhaltens sind Geschlechterunterschiede eher auf die jüngeren Altersgruppen beschränkt.

Für die Einschätzung, ob die Straftaten in Deutschland zugenommen haben, ist dagegen nur ein schwacher Alterseffekt erkennbar. Frauen sind häufiger als Männer der Ansicht, dass Straftaten ansteigen würden, in allen Altersgruppen. Eine Ausnahme betrifft die jüngsten Befragten: Bei den 16- bis 29-Jährigen sind Frauen und Männer gleichermaßen der Ansicht, dass es in Deutschland in den letzten zehn Jahren zu einer Zunahme der Kriminalität gekommen wäre.

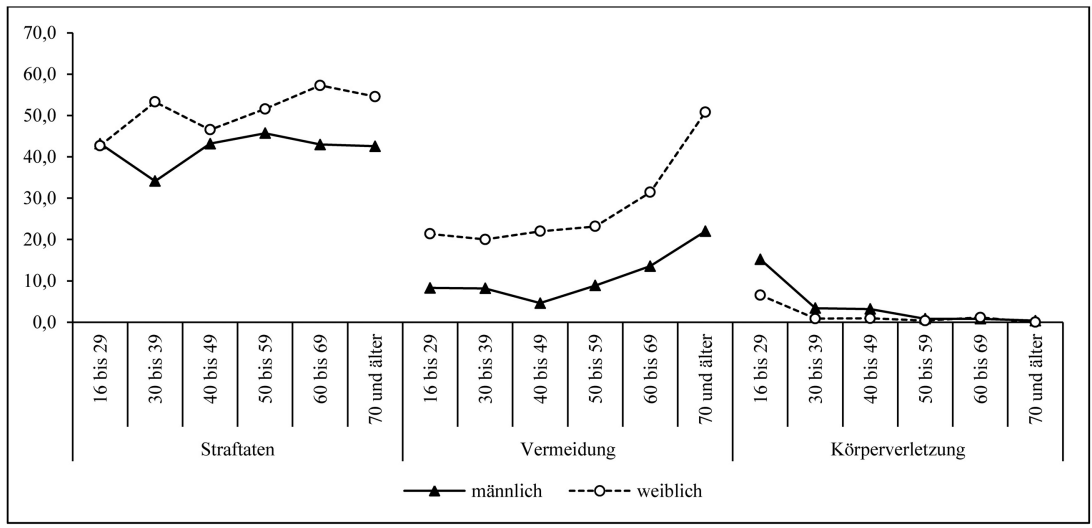

Abbildung 7. Wahrgenommene Kriminalitätsentwicklung, Kriminalitätsfurcht und Opferrate Körperverletzung nach Geschlecht und Altersgruppe (in \% gewichtete Daten).

\section{Diskussion}

Unter Rückgriff auf verschiedene Datenquellen sollten in diesem Beitrag zwei Fragen beantwortet werden. Erstens ging es darum, das Ausmaß der Geschlechterunterschiede für unterschiedliche Delikte und Personengruppen zu beschreiben. Zweitens war zu eruieren, ob es die Entwicklung von Geschlechterunterschieden erlaubt, auch im Bereich der Kriminalität von einer „Krise der Jungen“ zu sprechen. 
Hinsichtlich der ersten Frage kann festgehalten werden, dass es bei den meisten betrachteten Delikten Geschlechterunterschiede gibt. Vor allem im Bereich des Gewaltverhaltens bzw. der Gewaltviktimisierung zeigt sich, dass Männer höher belastet sind als Frauen. Eine Ausnahme ist der Bereich der sexuellen Gewalt, von der Frauen deutlich stärker betroffen sind als Männer. Im Bereich der Eigentumsdelikte gilt, dass der Diebstahl - betrachtet wurde vor allem der Ladendiebstahl - ein Deliktsbereich mit eher geringen Geschlechterunterschieden darstellt. Ladendiebstähle werden von weiblichen Jugendlichen laut Polizeilicher Kriminalstatistik häufiger, laut Dunkelfeldbefragungen fast genauso häufig ausgeführt wie von männlichen Jugendlichen. Bei den Sachbeschädigungen ist hingegen der bekannte gender gap mit höherer Belastung bei den Jungen festzustellen.

Zeigt bereits der Blick auf den Ladendiebstahl, dass der Geschlechterunterschied keine unverrückbare Konstante darstellt, belegt auch der Blick auf zwei weitere Bereiche, dass Frauen Auffälligkeiten zeigen. Erstens ist hier der Bereich der indirekten Aggressionen zu nennen. Verbale Angriffe erfolgen in sporadischer Form von weiblichen Jugendlichen ähnlich häufig wie von männlichen Jugendlichen. Die relationale Aggression, d. h. die Manipulation sozialer Beziehungen erfolgt durch Mädchen sogar häufiger. Mädchen schädigen mithin eher nicht direkt und körperlich, sondern eher indirekt und psychisch. Darüber hinaus ist zweitens für Frauen Kriminalität ein Thema, das ihnen mehr Sorgen bereitet. Frauen haben im Vergleich zu Männern mehr Furcht, Opfer krimineller Übergriffe zu werden, zeigen deshalb mehr Vermeidungsverhalten und gehen häufiger davon aus, dass Kriminalität in Deutschland ansteigt. Diese Wahrnehmungen stehen im Widerspruch zur Realität, da Kriminalität in Deutschland in verschiedenen Bereichen rückläufig ist und da Frauen seltener viktimisiert werden.

Im Vergleich verschiedener Bevölkerungsgruppen ergeben sich ebenfalls interessante Befunde zum gender gap. In Bezug auf das Gewaltverhalten gilt bspw., dass mit dem Älterwerden der Geschlechterunterschied zurückgeht. Körperverletzungen erleben laut Dunkelfeldbefunden ältere Frauen vergleichbar häufig wie Männer. Zudem gibt es eine weitere Gruppe an Personen, in der Geschlechterunterschiede vernachlässigbar sind. Männliche wie weibliche Strafgefangene erleben bzw. begehen in vergleichbarer Häufigkeit Gewalt. Gerade dieser letzte Befund weist darauf hin, dass es Bedingungen gibt, unter denen Frauen zur physischen Gewalt greifen. Hierbei sind sowohl personenbezogene Faktoren als auch situative Faktoren zu betrachten. Die personenbezogenen Faktoren betreffen die Eigenschaften wie eine erhöhte Aggressivität oder eine verringerte Selbstkontrolle, die dazu geführt haben, dass die Frauen straffällig gewor- 
den sind. Die situativen Faktoren betreffen die Besonderheiten des Strafvollzugs. Hier kommen Personen mit diesen problematischen Eigenschaften zusammen. Es wird damit ein Klima geschaffen, in dem die physische Gewalt ein zentrales Mittel des Umgangs mit anderen, des individuellen Statuserhalts und -gewinns ist; auch Frauen greifen unter diesen Bedingungen zu körperlicher Gewalt.

Hinsichtlich der zweiten Frage, ob eine „Krise der Jungen“ auch im Bereich der Kriminalität identifizierbar ist, sind die Befunde mehrdeutig. Eine Krise ließe sich paradigmatisch an einer Entwicklung festmachen, die in Bezug auf die Jungen bzw. Männer zu einem Anstieg, in Bezug auf die Mädchen bzw. Frauen zu einem Rückgang führt. Eine solche Entwicklung zeigt sich im Hell- wie im Dunkelfeld nur ein einziges Mal: für die Vergewaltigungen. Diese nehmen bei Männern zu, bei Frauen ab. Allerdings bleibt weiterhin eine deutlich höhere Viktimisierungsrate bei den Frauen bestehen. Zugleich dürfte diese Entwicklung weniger für eine Krise der Männer, als für eine Öffnung derselben stehen. Die Bereitschaft, entsprechende Übergriffe der Polizei zu berichten oder in Dunkelfeldbefragungen anzugeben, dürfte gestiegen sein. Für die Frauen dürfte die Entwicklung hingegen für eine tatsächliche, positive Veränderung stehen.

Eine „Krise der Jungen“ lässt sich an verschiedenen Stellen aber durchaus in anderer Weise identifizieren, nämlich derart, dass für Jungen ein geringerer Rückgang des kriminellen Verhaltens festzustellen ist als für Mädchen. Vor allem Dunkelfeldbefunde machen hierauf aufmerksam: Bei den Jugendlichen geht der Raub oder die Sachbeschädigung weniger deutlich bei den Jungen als beiden Mädchen zurück; bei den Erwachsenen findet sich dieses Muster ebenfalls beim Raub. Diese Veränderungen sind deshalb im Hellfeld nicht zu identifizieren, da die Anzeigequote geschlechtsspezifisch steigt. Dies konnte zumindest für Jugendliche gezeigt werden: Weibliche Gewalttäter werden heute deutlich häufiger angezeigt als früher. Dies könnte dahingehend interpretiert werden, dass sich die soziale Kontrolle stärker gegenüber Frauen als gegenüber Männern verändert. Wenn sich Mädchen nicht der Norm entsprechend verhalten, erfolgt auf dieses Verhalten eher eine Sanktion als wenn Jungen dies tun. Dies wäre eine Dimension der „Krise“: Das soziale Umfeld signalisiert nicht gleichermaßen stark Jungen und Mädchen, dass sich gesetzestreu zu verhalten ist, womit es Jungen noch häufiger zum Begehen von Straftaten ermuntert.

Es ist zugleich nicht angemessen, die Entwicklung im Bereich des kriminellen Verhaltens für Jungen nur negativ im Sinne einer „Krise“ zu betrachten. Die Auswertungen konnten mehrfach belegen, dass für die Ge- 
schlechter gleichartige positive Veränderungen stattfinden. Körperverletzungen nehmen, zumindest in kurzfristiger Betrachtung, über die Jahre bei Jugendlichen wie bei Erwachsenen ab. Der Mord/Totschlag geht deutlich zurück, ebenso der sexuelle Kindesmissbrauch oder der Diebstahl bzw. der Wohnungseinbruch. Auch kriminalitätsbezogene Wahrnehmungen und Einstellungen entwickeln sich für beide Geschlechter zum Positiven.

Grundsätzlich belegen die Auswertungen für Deutschland damit für verschiedene Bereiche einen Rückgang der Kriminalität. Zugleich bleiben einige Problembereiche bestehen, die eher die Eigentums- als die Gewaltkriminalität betreffen. Warum trotz der positiven Entwicklungen die Forderung nach harten Strafen weiterhin sehr weit verbreitet ist, sollte über weitere Studien, die sich u. a. der Kriminalitätsberichterstattung in den Medien widmen, der Quelle also, aus der die meisten Menschen ihr Wissen über Kriminalität ziehen, geklärt werden.

\section{Literatur}

Baier, D. (2008). Entwicklung der Jugenddelinquenz und ausgewählter Bedingungsfaktoren seit 1998 in den Städten Hannover, München, Stuttgart und Schwäbisch Gmünd (Forschungsbericht No. 104). Hannover: Kriminologisches Forschungsinstitut Niedersachsen.

Baier, D. (2011a). Jugendgewalt im Landkreis Soltau Fallingbostel - Ergebnisse einer Wiederholungsbefragung. (Forschungsbericht No. 116). Hannover: Kriminologisches Forschungsinstitut Niedersachsen.

Baier, D. (2011b). Jugendgewalt in Deutschland - Eine Bestandsaufnahme. In G. Deegener \& W. Körner (Hrsg.), Gewalt und Aggression im Kindes- und Jugendalter. Ursachen, Formen, Intervention (S. 35-53). Weinheim: Beltz.

Baier, D. (2011c). Jugendgewalt und Geschlecht - Erkenntnisse aus Kriminalstatistik und Dunkelfelduntersuchungen. Zeitschrift für Jugendkriminalrecht und Jugendhilfe, 22, 356-364.

Baier, D. \& Bergmann, M. C. (2013). Gewalt im Strafvollzug - Ergebnisse einer Befragung in fünf Bundesländern. Forum Strafvollzug, 62, 76-83.

Baier, D. \& Branig, C. (2009). Educational Expansion and Delinquency. In A. Hadjar $\&$ R. Becker (Hrsg.), Expected and Unexpected Consequences of the Educational Expansion in Europe and the US (S. 349-359). Bern: Haupt Verlag.

Baier, D., Kemme, S., Hanslmaier, M., Doering, B., Rehbein, F. \& Pfeiffer, C. (2011). Kriminalitätsfurcht, Strafbedürfnisse und wahrgenommene Kriminalitätsentwicklung. Ergebnisse von bevölkerungsrepräsentativen Befragungen aus den Jahren 2004, 2006 und 2010 (Forschungsbericht No. 117). Hannover: Kriminologisches Forschungsinstitut Niedersachsen. 
Baier, D., Pfeiffer, C., Simonson, J. \& Rabold, S. (2009). Jugendliche in Deutschland als Opfer und Täter von Gewalt. Erster Forschungsbericht zum gemeinsamen Forschungsprojekt des Bundesministerium des Innern und des KFN (Forschungsbericht No. 107). Hannover: Kriminologisches Forschungsinstitut Niedersachsen.

Baier, D., Pfeiffer, C., Windzio, M. \& Rabold, S. (2006). Schülerbefragung 2005: Gewalterfahrungen. Schulabsentismus und Medienkonsum von Kindern und Jugendlichen. Abschlussbericht über eine repräsentative Befragung von Schülerinnen und Schülern der 4. und 9. Jahrgangsstufe. Hannover: Kriminologisches Forschungsinstitut Niedersachsen. Verfügbar unter: http://www.kfn.de/Forschungsbereiche_und_Projekte/Schuelerbefragungen/Schuelerbefragung_2005.htm.

Bilsky, W., Mecklenburg, E. \& Wetzels, P. (1992). Persönliches Sicherheitsgefühl, Angst vor Kriminalität und Gewalt, Opfererfahrung älterer Menschen (Forschungsbericht No. 13). Hannover: Kriminologisches Forschungsinstitut Niedersachsen.

Dünkel, F., Gebauer, D. \& Geng, B. (2007). Gewalterfahrungen, gesellschaftliche Orientierungen und Risikofaktoren von Jugendlichen in der Universitäts- und Hansestadt Greifswald 1998-2002-2006: Universität Greifswald.

Ellrich, K. (2012). Gewalt gegen Polizeibeamte. In C. Lorei \& J. Sohnemann (Hrsg.), Grundwissen Eigensicherung (S. 5-34). Frankfurt: Verlag für Polizeiwissenschaft.

Ellrich, K., Baier, D. \& Pfeiffer, C. (2012). Polizeibeamte als Opfer von Gewalt. Ergbnisse einer Befragung von Polizeibeamten in zehn Bundesländern. BadenBaden: Nomos.

Ernst, S. (2008). Gewalt unter erwachsenen männlichen Inhaftierten in deutschen Justizvollzugsanstalten. Hamburg: Verlag Dr. Kovac.

Heinrich, W. (2002). Gewalt im Gefängnis - eine Untersuchung der Entwicklung von Gewalt im hessischen Justizvollzug (1989-1998). Bewährungshilfe, 49, 369-383.

Heinz, W., Spieß, G., Schnell, R. \& Kreuter, F. (1998). Opferbefragung 1997. Bericht für das Bundesministerium der Justiz. Konstanz: Bundesministerium der Justiz.

Junger-Tas, J., Ribeaud, D. \& Cruyff, M. J. L. F. (2004). Juvenile Delinquency and Gender. European Journal of Criminology, 1, 333-375.

Kunz, F. (2011). Kriminelles Verhalten und polizeiliche Registrierung. Selbstberichte im höheren Lebensalter. Zeitschrift für Gerontologie und Geriatrie, 1, 55-65.

Kury, H. (1992). Opfererfahrungen und Meinungen zur Inneren Sicherheit in Deutschland. Wiesbaden: BKA.

Lamnek, S. (1998). Kriminalität. In B. Schäfers \& W. Zapf (Hrsg.), Handwörterbuch zur Gesellschaft Deutschlands (Bd. 382-393). Opladen: Leske und Budrichs.

Mehlkop, G. (2011). Kriminalität als rationale Wahrnehmung. Wiesbaden: VS Verlag.

Rabold, S. (2014). Ziele und Methoden der Dunkelfeldforschung. Manuskript im Druck.

Rabold, S., Baier, D. \& Pfeiffer, C. (2008). Jugendgewalt und Jugenddelinquenz in Hannover (Forschungsberichte No. 105). Hannover: Kriminologisches Forschungsinstitut Niedersachsen.

Scheithauer, H. (2003). Aggressives Verhalten von Jungen und Mädchen. Göttingen: Hogrefe. 
Stadler, L., Bieneck, S. \& Pfeiffer, C. (2012). Repräsentativbefragung sexueller Missbrauch 2011 (Forschungsbericht No. 118). Hannover: Kriminologisches Forschungsinstitut Niedersachsen.

Wetzels, P. (1997). Gewalterfahrungen in der Kindheit. Sexueller Mißbrauch, körperliche Mißhandlung und deren langfristige Konsequenzen. Baden-Baden: Nomos.

Wetzels, P., Enzmann, D., Mecklenburg, E. \& Pfeiffer, C. (2001). Jugend und Gewalt. Eine repräsentative Dunkelfeldanalyse in München und acht anderen deutschen Städten. Baden-Baden: Nomos.

Wirth, W. (2007). Gewalt unter Gefangenen. Kernbefunde einer empirischen Studie im Strafvollzug des Landes Nordrhein-Westfalen. Bewährungshilfe, 54, 185-206. 


\section{Mögliche Erklärungen beobachteter Geschlechterunterschiede}


